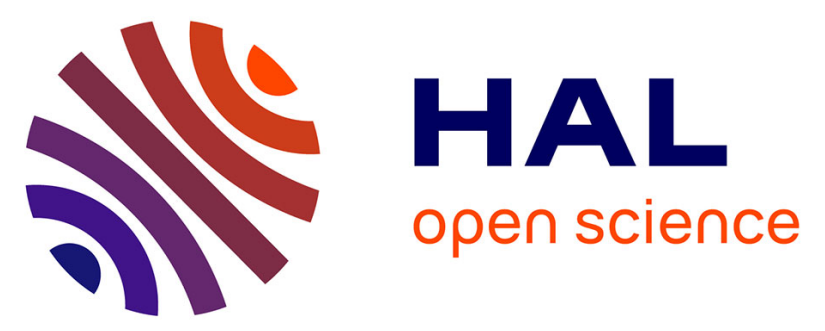

\title{
Forward scattering and filamentation of a spatially smoothed laser pulse in the hydrodynamic and kinetic frameworks
}

$\ddagger$ M Nakatsutsumi, C. Ruyer, A. Debayle, P. Loiseau, P. E Masson-Laborde, J. Fuchs, M. Casanova, J. R Marquès, L. Romagnani, P. Antici, et al.

\section{To cite this version:}

$\ddagger$ M Nakatsutsumi, C. Ruyer, A. Debayle, P. Loiseau, P. E Masson-Laborde, et al.. Forward scattering and filamentation of a spatially smoothed laser pulse in the hydrodynamic and kinetic frameworks. Physics of Plasmas, 2021, 28 (5), pp.052701. 10.1063/5.0043931 . hal-03355313

\section{HAL Id: hal-03355313 \\ https://hal.science/hal-03355313}

Submitted on 27 Sep 2021

HAL is a multi-disciplinary open access archive for the deposit and dissemination of scientific research documents, whether they are published or not. The documents may come from teaching and research institutions in France or abroad, or from public or private research centers.
L'archive ouverte pluridisciplinaire HAL, est destinée au dépôt et à la diffusion de documents scientifiques de niveau recherche, publiés ou non, émanant des établissements d'enseignement et de recherche français ou étrangers, des laboratoires publics ou privés. 


\title{
Forward scattering of a plane wave and of a spatially smoothed laser pulse in the hydrodynamic and kinetic frameworks
}

\author{
C. Ruyer, ${ }^{1,2, *}$ A. Debayle, ${ }^{1,2}$ M. Casanova,${ }^{1}$ P. Loiseau,,${ }^{1,2}$ P. E. Masson-Laborde,${ }^{1,2}$ J. Fuchs,${ }^{3}$ J. R. Marquès,${ }^{3}$ L. \\ Romagnani, ${ }^{3}$ P. Antici, ${ }^{3, \dagger}$ N. Bourgeois,${ }^{3,}$ M. Nakatsutsumi, ${ }^{4,}$ M. Safronova, ${ }^{5}$ M. Starodubtsev, ${ }^{5}$ and T. Lin $^{6}$ \\ ${ }^{1}$ CEA, DAM, DIF, F-91297 Arpajon, France \\ ${ }^{2}$ Université Paris-Saclay, CEA, LMCE, 91680 Bruyères-le-Châtel, France \\ ${ }^{3}$ LULI - CNRS, CEA, UPMC Univ Paris 06: Sorbonne Université, \\ Ecole Polytechnique, Institut Polytechnique de Paris - F-91128 Palaiseau Cedex, France \\ ${ }^{4}$ Institute of Laser Engineering, Osaka University, \\ 02-06 Yamada-Oka, Suita, Osaka, 565-0871, Japan \\ ${ }^{5}$ Institute of Applied Physics, RAS, 46 Ulyanov Street, 603950 Nizhny Novgorod, Russia \\ ${ }^{6}$ Fox Chase Cancer Center, 333 Cottman, Philadelphia, PA 19111-2497, USA
}

\begin{abstract}
We address the scattering of a high energy laser pulse on a large wavelength acoustic turbulence of relevance for LMJ or NIF-class experiments. Both kinetic and hydrodynamic frameworks are adopted and combined with a linearized description of the laser propagation. The resulting dispersion relations display important kinetic contributions to the growth of the Forward Brillouin instability. Moreover, proof is made that the spatial incoherence often used in high energy laser facilities are, for cold enough plasmas or in the multi-ion species case, not enough to reach full control of the laser filamentation. Comparisons with experimental results and dedicated hydrodynamic simulations confirm our results. The derived dispersion relations present unique tools for assessing the propagation quality and energy deposition region of high energy laser pulses. They also underline the importance of accounting correctly for kinetic effects, even in the millimeters and nanoseconds scale of many ICF or high-energy-density experiments.
\end{abstract}

\section{INTRODUCTION}

High energy class laser facilities such as LMJ, NIF or SG-III routinely bring matter under extreme conditions, not only of interest for inertial confinement fusion (ICF) [1-3], but also for high energy astrophysics and high energy density physics [4]. Whether the laser serves as a heat source or as a diagnostic, the accurate prediction of its propagation remains crucial for designing and conceiving experiments. To this end, a large effort is made for understanding and mitigating the wave mixing processes able to scatter a significant part of the electromagnetic energy towards unwanted directions $[5,6]$. A better control of the laser propagation has been achieved experimentally by the use of Spectral Dispersion (SSD) and Random Phase Plates (RPP) optical smoothing techniques[7-10] and have been considered efficient to mitigate filamentation instability [1114], beam bending[15-18] and forward Brillouin. To date, only stimulated backward Raman or Brillouin instabilities [19, 20], cross beam energy transfer [21], two plasmon decay $[22,23]$ or collective scattering [24, 25], are identified as critical issues in ICF, and remain active area of research. However, the small mitigation of cross beam

\footnotetext{
* charles.ruyer@cea.fr

$\dagger$ present address: INRS-EMT, 1650, boulevard Lionel-Boulet, J3X 1S2, Varennes (Québec), Canada

¥ present address: Central Laser Facility, STFC Rutherford Appleton Laboratory, Didcot OX11 0QX, United Kingdom

$\S$ present address: European XFEL, GmbH, Holzkoppel 4, 22869 Schenefeld, Germany
}

energy transfer [26] and backward Brillouin scattering by laser smoothing techniques may presage similar tendencies concerning the forward scattering. The latter can significantly alter the laser beam propagation by increasing the beam opening angle, thereby explaining the small laser beam transmission measured in some experiments [27].

To date, hydrodynamic codes remain the main tools to describe most of ICF experiments, due to their relatively affordable numerical cost. A first class of hydrodynamic codes, able to resolve the speckle scale structure, can describe different wave-mixing processes on a reduced plasma using regular meshes [28-31]. However, the Landau damping rate which is the main parameter of wave-mixing processes in plasma, is reduced to an ad hoc correction only valid in weakly-damped single-ion species plasma. Moreover, the non-local electron heat transport, thought to be important between each speckles during the heating phase [32, 33], is usually neglected. The second class of hydrodynamic codes, able to describe full scale ICF experiments on unstructured meshes, further neglects the speckle scale structure. The laser beam propagation is reduced to a ray-tracing scheme in which most of wave mixing processes are omitted. Recently, a ray-tracing algorithm has been proposed [34] to selfconsistently describe the cross beam energy transfer, the Raman and Brillouin backward scatterings. In principle, adding the convective growth of the filamentation and the forward Brillouin scattering are straightforward. However, this scheme neglects the speckle scale structure and assumes linear convective rates of wave-mixing processes based on a plane wave electromagnetic field. Alas, the resulting micron-scale speckle dynamics that 
interweaves with the wave mixing processes, brings further complexity to the theoretical and numerical analysis [35]. Building a self-consistent set of equations, adapted for Lagrangian hydrodynamic codes, that properly encompasses the filamentation instability, forward Brillouin scattering and plasma smoothing effects [36-38], remains a challenging task.

This publication ambitions are to convey new tools able to address the spatial growth of an electromagnetic scattered wave of wavevector and frequency close to the RPP pulse's and embedded in driven acoustic density fluctuations. We first derive both the kinetic and fluid forward scattering of a plane wave, limiting the study to the spatial growth of stimulated forward Brillouin scattering (FSBS) and the filamentation instabilities and pinpoint the importance of accounting for the kinetic plasma response for understanding quantitatively the scattering growth. From a simple model of RPP smoothed laser beam ensues our dispersion relations able to predict the growth of the forward scatter relevant for high energy laser experiments. A comparison with dedicated hydrodynamic simulations and with an experiment conducted at the LULI facility validate our predictions. The last section gather our perspective and concluding remarks.

The SI unit system is used throughout this study while dropping the Boltzmann constant and vectors are noted in bold symbols.

\section{SPATIAL GROWTH OF THE FORWARD SCATTERING OF A PLANE WAVE}

\section{A. Fluid and kinetic general dispersion relation}

The simplest way to obtain the growth of the forward scattering of a light wave propagating in a plasma consists in combining the perturbed Maxwell equations with a linearized plasma response, either kinetic or fluid. We aim at deriving and comparing in this section both frameworks.

Following Refs. [11, 39], the perturbation of Maxwell equations around the laser field, $E_{l}$, i.e. $E=E_{l}+\delta E$ and around the electron density at rest $n_{e}=n_{e 0}+\delta n_{e}$, gives in the Fourier space $\left(\omega_{d}, \mathbf{k}_{d}\right)$

$$
\left(\omega_{d}^{2}-\omega_{p e}^{2}-\mathbf{k}_{d}^{2} c^{2}\right) \delta E\left(\omega_{d}, \mathbf{k}_{d}\right)=\omega_{0}^{2} \frac{\delta n_{e}}{n_{c}} \otimes E_{l},
$$

where $\omega_{p e}, \omega_{0}, n_{c}$ and $c$ are the plasma and laser frequency, laser critical density and light speed in vacuum respectively. Use has also been made of $\otimes$ which designates here a convolution product in the Fourier space. Within the plane wave approximation, $E_{l}=E_{0} \cos \left(k_{0} x-\right.$ $\left.\omega_{0} t\right)$, and we may write in the Fourier space,

$$
\begin{array}{r}
\mathrm{FT}_{\omega, \mathbf{k}}\left[E_{0} \cos \left(k_{0} x-\omega_{0} t\right)\right]= \\
{\left[\delta\left(\omega-\omega_{0}, \mathbf{k}-\mathbf{k}_{0}\right)+\delta\left(\omega+\omega_{0}, \mathbf{k}+\mathbf{k}_{0}\right)\right] E_{0} / 2}
\end{array}
$$

where $\mathrm{FT}[f(x, t)]$ designates the Fourier transform in space and time as $\frac{1}{(2 \pi)^{2}} \int f(x, t) e^{-i k x+i \omega t} d x d t$. In this section, $\mathbf{k}_{0}=k_{0} \hat{\mathbf{x}}$ is the $x$-aligned pump wavevector $(\hat{\mathbf{x}}$ is the unity vector along the $x$ axis), thus giving

$$
\begin{array}{r}
\left(\omega_{d}^{2}-\omega_{p e}^{2}-\mathbf{k}_{d}^{2} c^{2}\right) \delta E\left(\omega_{d}, \mathbf{k}_{d}\right)=\frac{\omega_{0}^{2}}{2} E_{0} \times \\
{\left[\frac{\delta n_{e}}{n_{c}}\left(\omega_{d}-\omega_{0}, \mathbf{k}_{d}-\mathbf{k}_{0}\right)+\frac{\delta n_{e}}{n_{c}}\left(\omega_{d}+\omega_{0}, \mathbf{k}_{d}+\mathbf{k}_{0}\right)\right]}
\end{array}
$$

The final dispersion relation can be obtained by combining this equation with a linearized plasma response, which, in the fluid framework [18] and for a plasma at rest, reads

$$
\begin{array}{r}
\frac{\delta n_{e}}{n_{e 0}}\left(\omega_{s}, \mathbf{k}_{s}\right)=\frac{-\kappa \mathbf{k}^{2} c_{s}^{2}}{\mathbf{k}_{s}^{2} c_{s}^{2}-\omega_{s}^{2}-2 i \omega_{s} \nu} \frac{A_{k} \epsilon_{0} E_{0}}{n_{c} T_{e}} \times \\
{\left[\delta E\left(\omega_{s}-\omega_{0}, \mathbf{k}_{s}-\mathbf{k}_{0}\right)+\delta E\left(\omega_{s}+\omega_{0}, \mathbf{k}_{s}+\mathbf{k}_{0}\right)\right] .}
\end{array}
$$

Here, $\kappa=Z_{i} T_{e} / m_{i} c_{s}^{2}, c_{s} \simeq\left[\left(Z_{i} T_{e}+3 T_{i}\right) / m_{i}\right]^{1 / 2}, \nu=$ $\left|\mathbf{k}_{s}\right| \gamma_{0} c_{s}$ and $A_{k}$, are respectively the sound speed, the Landau damping frequency and a non-local thermal correction to the ponderomotive force[40],

$$
\begin{aligned}
A_{k}(u) & =\frac{1}{2}+Z_{i}\left(\frac{0.074}{u^{2}}+\frac{0.88}{u^{4 / 7}}+\frac{2.54}{1+5.5 u^{2}}\right), \\
u & =\left|\mathbf{k}_{s}\right| \lambda_{\operatorname{mfp}} \sqrt{Z_{i}} .
\end{aligned}
$$

We also made use of $T_{e / i}, m_{e / i} Z_{i}$ and $\lambda_{\text {mfp }}$, the electron/ion temperature and mass, ion charge number and electron mean-free-path, respectively. For a multi-ion species plasma, the smallest mean-free-path among the ion species is used to compute $A_{k}$. The correction of Ref. [40] requires to account for the collisional acoustic damping rate which is negligible for the plasma parameters addressed here $\left(T_{e} \gtrsim 1 \mathrm{keV}, n_{e} \leq 10^{21} \mathrm{~cm}^{-3}\right.$ and $I_{0} \leq 10^{15} \mathrm{~W} / \mathrm{cm}^{2}$ ) or irrelevant (as for the filamentation instability, Secs. III B and III C). Note that, for the parameters explored in this study, the non-local correction remains non negligible and therefore essential to obtain realistic predictions $[41,42]$.

In the following, the normalized acoustic wave Landau damping rate, $\gamma_{0}$,

$$
\begin{aligned}
\gamma_{0} & =\sqrt{\frac{2 T_{i}}{m_{i} c_{s}^{2}} \Im\left[\frac{\mathcal{Z}^{\prime}\left(x_{i}\right)+\frac{T_{i}}{Z_{i} T_{e}} \mathcal{Z}^{\prime}\left(x_{e}\right)}{\mathcal{Z}^{\prime \prime}\left(x_{i}\right)+\left(\frac{T_{i}}{Z_{i} T_{e}}\right)^{3 / 2} \sqrt{\frac{Z_{i} m_{e}}{m_{i}}} \mathcal{Z}^{\prime \prime}\left(x_{e}\right)}\right]} \\
x_{e / i} & =\sqrt{\frac{m_{e / i} c_{s}^{2}}{2 T_{e / i}}}
\end{aligned}
$$

will be used under the assumptions $\nu /\left|\mathbf{k}_{\perp}\right| c_{s}=\gamma_{0} \ll 1$, $\left|\mathbf{k}_{\perp}\right| \lambda_{D i} \ll 1 . \lambda_{D e / i}$ is the electron/ion Debye length and $\mathcal{Z}$ is the plasma dispersion function [43].

Likewise, the kinetic counterpart of Eq. (4) has been derived in Ref. [44] and prevails in the case of a multiple 
ion Maxwellian plasma. It writes

$$
\begin{array}{r}
\frac{\delta n\left(\omega_{s}, \mathbf{k}_{s}\right)}{n_{e 0}}=\frac{\epsilon_{0} E_{0}}{2 n_{c} T_{e}} \frac{\mathcal{Z}^{\prime}\left(\zeta_{e}\right)}{2} \frac{\sum_{i} \mathcal{Z}^{\prime}\left(\zeta_{i}\right) \frac{Z_{i} T_{e}}{T_{i}} \frac{Z_{i} n_{i}}{n_{e}}}{\mathcal{Z}^{\prime}\left(\zeta_{e}\right)+\sum_{i} \mathcal{Z}^{\prime}\left(\zeta_{i}\right) \frac{Z_{i} T_{e}}{T_{i}} \frac{Z_{i} n_{i}}{n_{e}}} \\
\times\left[\delta E\left(\omega_{s}-\omega_{0}, \mathbf{k}_{s}-\mathbf{k}_{0}\right)+\delta E\left(\omega_{s}+\omega_{0}, \mathbf{k}_{s}+\mathbf{k}_{0}\right)\right] \\
(7) \\
\zeta_{e / i}=\frac{\omega}{\left|\mathbf{k}_{s}\right|} \sqrt{\frac{m_{e / i}}{2 T_{e / i}}},
\end{array}
$$

where we also assumed $\left|k_{s} \lambda_{D i}\right| \ll 1$.

To unify the fluid and kinetic descriptions, we introduce the parameters

$$
\begin{array}{r}
\alpha_{k}=\frac{-\mathcal{Z}^{\prime}\left(\zeta_{e}\right)}{2} \frac{\sum_{i} \mathcal{Z}^{\prime}\left(\zeta_{i}\right) \frac{Z_{i} T_{e}}{T_{i}} \frac{Z_{i} n_{i}}{n_{e}}}{\mathcal{Z}^{\prime}\left(\zeta_{e}\right)+\sum_{i} \mathcal{Z}^{\prime}\left(\zeta_{i}\right) \frac{Z_{i} T_{e}}{T_{i}} \frac{Z_{i} n_{i}}{n_{e}}}, \\
\alpha_{f}=\frac{\kappa c_{s}^{2}}{c_{s}^{2}-v_{\phi}^{2}-2 i v_{\phi} c_{s} \gamma_{0}}
\end{array}
$$

where $v_{\phi}=\omega_{s} /\left|\mathbf{k}_{s}\right|$ is the phase velocity. Both Eqs. (4) and (7) recast as:

$$
\begin{array}{r}
\frac{\delta n_{e}}{n_{e 0}}\left(\omega_{s}, \mathbf{k}_{s}\right)=-\alpha_{k / f}\left(v_{\phi}\right) \frac{A_{k} \epsilon_{0} E_{0}}{n_{c} T_{e}} \times \\
{\left[\delta E\left(\omega_{s}-\omega_{0}, \mathbf{k}_{s}-\mathbf{k}_{0}\right)+\delta E\left(\omega_{s}+\omega_{0}, \mathbf{k}_{s}+\mathbf{k}_{0}\right)\right]}
\end{array}
$$

Note that we have applied the non-local correction [Eq. (5)] in the kinetic framework, as there is, to the best of our knowledge, no better simple alternative. Originally derived in the fluid framework, its proper kinetic counterpart would require to start from Refs. [45, 46] and is out of the scope of the present study. As the $A_{k}$-factor is relevant in the fluid framework, choice has been made to include it in the kinetic formalism for comparison purposes. Yet, its use is questionable regarding the forward Brillouin predictions.

From the combination of Eqs. (3) with (11), for $D_{ \pm}=$ $\left(\omega_{s} \pm \omega_{0}\right)^{2}-\omega_{p e}^{2}-\left(\mathbf{k}_{s} \pm \mathbf{k}_{0}\right)^{2} c^{2}$, ensues

$$
D_{+} D_{-}=-\omega_{0}^{2} \alpha_{k / f} A_{k} \frac{\delta n_{0}}{n_{c}}\left(D_{+}+D_{-}\right)
$$

with $\delta n_{0} / n_{c}=\left(n_{e 0} / n_{c}\right) \epsilon_{0} E_{0}^{2} /\left(2 n_{c} T_{e}\right)$. Note that we neglected the terms in $\delta n_{e}\left(\omega_{s} \pm 2 \omega_{0}\right)$. In the kinetic framework, the above equation corresponds to Eq. (36) of Ref. [47] in the limit $\left|k_{s} \lambda_{D i}\right| \ll 1$. In the fluid framework, similar equations were derived in Refs. [11, 36, 48].

\section{B. Spatial growth of the filamentation and of the forward Brillouin instability}

Hence, making use of $\omega_{0}^{2}=\omega_{p e}^{2}+k_{0}^{2} c^{2}$, we may simplify $D_{ \pm}$to leading order in $\omega_{s} \ll \omega_{0}$, giving

$$
D_{ \pm}=-\mathbf{k}_{s}^{2} c^{2} \pm 2\left(\omega_{s} \omega_{0}-\mathbf{k}_{s} \cdot \mathbf{k}_{0} c^{2}\right) .
$$

(a) Kinetic, $\Gamma / k_{0}$

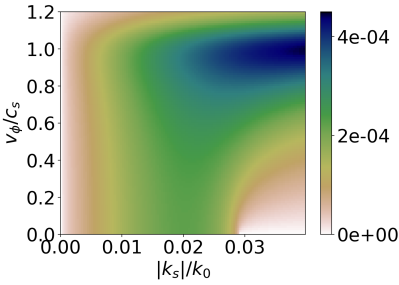

(c) Fluid, $\Gamma / k_{0}$

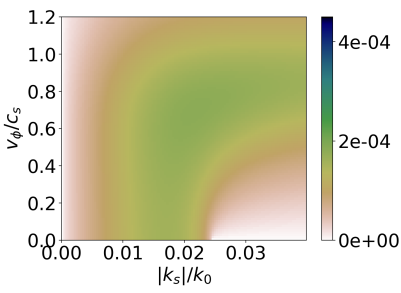

(b) Kinetic, $\Re\left(k_{s x} / k_{0}\right)$

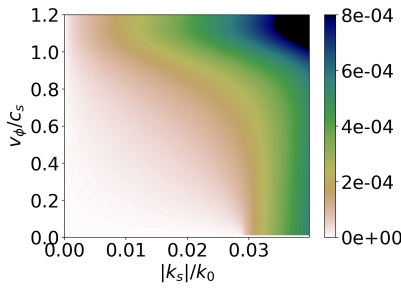

(d) Fluid, $\Re\left(k_{s x} / k_{0}\right)$

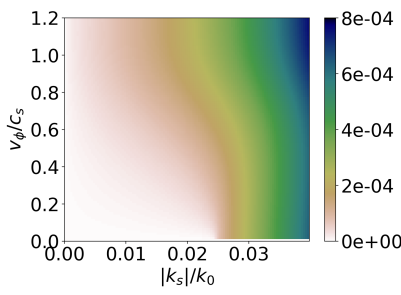

Figure 1. Kinetic (a,b) and fluid (c,d) resolution of Eq. (17) for $I_{0}=6 \cdot 10^{14}$ W.cm ${ }^{-2}, 2 \pi / k_{0}=0.35 \mu \mathrm{m}, T_{e}=1 \mathrm{keV}$, $T_{i}=300 \mathrm{eV}$ in $\mathrm{a} \mathrm{H}^{+}$and $n_{e 0}=0.1 n_{c}$.

Equation (12) thus becomes

$\left(\omega_{s} \omega_{0}-\mathbf{k}_{s} \cdot \mathbf{k}_{0} c^{2}\right)^{2}=\frac{\mathbf{k}_{s}^{2} c^{2}}{4}\left(\mathbf{k}_{s}^{2} c^{2}-2 \omega_{0}^{2} \alpha_{k / f}\left(v_{\phi}\right) A_{k} \frac{\delta n_{0}}{n_{c}}\right)$

The spatial growth of the filamentation instability may be recovered when $\omega_{s}=0$ and assuming $\mathbf{k}_{s}=-i \Gamma_{F} \hat{\mathbf{x}}+$ $k_{s} \hat{\mathbf{y}}$

$$
\Gamma_{F}^{2}=\frac{\mathbf{k}_{s}^{2}}{4 k_{0}^{2} c^{2}}\left(2 \omega_{0}^{2} \alpha_{k / f}(0) A_{k} \frac{\delta n_{0}}{n_{c}}-\mathbf{k}_{s}^{2} c^{2}\right) .
$$

Note that for a single ion species, $\alpha_{f}(0)=1$ and $\alpha_{k}(0)=$ $Z_{i} T_{e} / T_{i} /\left(1+Z_{i} T_{e} / T_{i}\right)$ so that the kinetic and fluid frameworks coincide in the limit $Z_{i} T_{e} / T_{i} \gg 1$.

As for the forward Brillouin scattering, corresponding to the limit $1 / D_{-} \gg 1 / D_{+}$, non vanishing acoustic wave frequencies are obtained with phase speeds of the order of the sound speed. We propose to address the spatial growth of both the filamentation and forward Brillouin instabilities by solving Eq. (14) for $\mathbf{k}_{s}=k_{s x} \hat{\mathbf{x}}+k_{s y} \hat{\mathbf{y}}$, and assuming $k_{s y}$ and $k_{s x}$ purely real and complex respectively. Equation (14) then becomes

$$
\left(\frac{v_{\phi}}{\eta c}-\frac{k_{s x}}{\left|\mathbf{k}_{s}\right|}\right)^{2}=\frac{1}{4}\left(\frac{\mathbf{k}_{s}^{2}}{k_{0}^{2}}-2 \alpha_{k / f}\left(v_{\phi}\right) A_{k} \frac{\delta n_{0}}{\eta^{2} n_{c}}\right),
$$

where $\eta=\sqrt{1-n_{e 0} / n_{c}}$ is the refraction index. Hence, $u=k_{s x} /\left|\mathbf{k}_{s}\right|$ is solution of the following second order polynomial equation,

$$
u^{2}-2 \frac{v_{\phi}}{\eta c} u+\frac{v_{\phi}^{2}}{\eta^{2} c^{2}}-\frac{1}{4}\left(\frac{\mathbf{k}_{s}^{2}}{k_{0}^{2}}-2 \alpha_{k / f}\left(v_{\phi}\right) A_{k} \frac{\delta n_{0}}{\eta^{2} n_{c}}\right)=0 .
$$

Figures $1(\mathrm{a}-\mathrm{d})$ display $\Re\left(k_{s x}\right)$ and the spatial growth rate, $\Gamma=-\Im\left(k_{s x}\right)$, combining the unstable parts of the 
(a) Kinetic, $\Gamma / k_{0}$

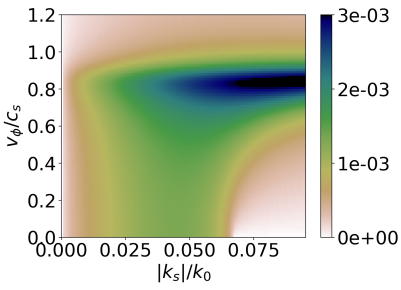

(c) Fluid, $\Gamma / k_{0}$

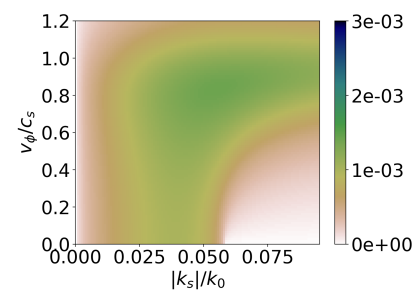

(b) Kinetic, $\Re\left(k_{s x} / k_{0}\right)$

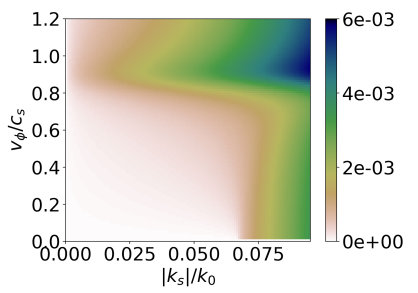

(d) Fluid, $\Re\left(k_{s x} / k_{0}\right)$

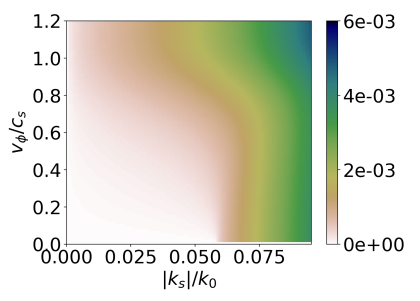

Figure 2. Kinetic (a,b) and fluid (c,d) resolution of Eq. (17) for $I_{0}=6 \cdot 10^{14} \mathrm{~W} . \mathrm{cm}^{-2}, 2 \pi / k_{0}=0.35 \mu \mathrm{m}$, for a CH plasma with $n_{c}=n_{H}, T_{e}=700 \mathrm{eV}, T_{C}=T_{H}=500 \mathrm{eV}$ and $n_{e 0}=$ $0.1 n_{c}$. The value of the non-local correction [Eq. (5)], is obtained with the carbon parameters, corresponding to the smallest electron-ion mean-free-path. Use is made of the mean charge number, mean mass number for calculating the sound speed and normalized Landau damping rate $c_{s}$ and $\gamma_{0}$.

two solutions of Eq. (17) as a function of the phase speed and of the wavevector amplitude, in both the kinetic $(a, b)$ and fluid frameworks $(\mathrm{c}, \mathrm{d})$. The filamentation limit is recovered at $v_{\phi}=0$ and care has been taken to verify that both frameworks coïncide, when $T_{i} \ll Z_{i} T_{e}$. Moreover, in both calculations, $\Re\left[k_{s x}\left(v_{\phi}=0\right)\right]$ is vanishing as expected from the filamentation instability. As $v_{\phi}$ increases, the forward Brillouin instability prevails and reaches its maximum, for the kinetic mono-ion species case of Fig. 1(a), around $\left(\left|k_{s}\right| / k_{0}, v_{\phi} / c_{s}\right) \simeq(0.03,1)$. Interestingly, only in the kinetic framework the forward Brillouin spatial maximum growth dominates the filamentation one, for the plasma parameters addressed here. In the fluid framework however both instability present a close growth rate of $\sim 1.5 \times 10^{-4} k_{0}$ with much broader spectrum than in the kinetic case.

The kinetic calculations allow to consider multiple ion plasmas such as $\mathrm{CH}$ as illustrated in Fig. 2. In the fluid framework, we are usually constrained to use the averaged-ion approximation, which gives qualitatively similar results than for Fig. 1(c) as a broad spectrum is evidenced with no dominance of the Brillouin versus the filamentation growth. This contrasts with the kinetic calculations of Fig. 2(a) where the FSBS is more unstable than the filamentation instability. Moreover the growth is peaked around $0.8 c_{s}$ (here $c_{s}$ is calculated on the averaged ion parameters) which is the phase speed of the least-damped acoustic eigenmode, solution of the free-field electrostatic Maxwellian dispersion relation [49, 50]. Notably, the forward Brillouin growth rate is twice larger in the kinetic formalism compared to the fluid description. This substantial difference, visible in both the hydrogen and $\mathrm{CH}$ plasma, vanishes in the case of the filamentation instability where both frameworks predict similar growth rates. This driven ion acoustic wave $\left(\left|v_{\phi}\right|>0\right)$ is able to scatter the pump wave and modify its spatial spectrum. As evidenced in Figs. 1(a,c) and $2(\mathrm{a}, \mathrm{c})$, we expect a broadening of the plane wave spatial spectrum resulting in an effective f-cone angle of $k_{s} / k_{0} \sim 0.03-0.05$, corresponding to $\sim 2-3^{\circ}$.

Spatial smoothing techniques are commonly known to restrain the role of some deleterious instabilities, such as the laser filamentation, during the propagation of energetic laser pulses $[7,9,51]$. Indeed, as shown in this section, the most unstable wavelength, regarding the filamentation or the forward Brillouin instabilities, is of the order of ten microns (for $\sim \mathrm{keV}$ and $10 \%$ critical density plasmas), thus larger than the typical speckle size of a few microns usually used in energetic laser facilities. Hence, the plane wave approximation used in obtaining Eq. (3) no longer holds and one might expect a more stable pump propagation. Yet, although extensively studied in the fluid framework by mean of numerical or theoretical tools [13, 36-38, 52-55], to the best of our knowledge, no analytical attempts were done to estimate the spatial growth of the forward Brillouin scattering of a RPP pulse in the kinetic framework. We propose in next section to adapt the above analytical plane wave dispersion relations to tackle this issue.

\section{FORWARD SCATTERING OF A SPATIALLY SMOOTHED LASER PULSE}

\section{A. Kinetic and fluid dispersion relations}

The RPP beam model adopted here has been introduced in Refs. [56, 57] and presents an electric field, $E_{\mathrm{RPP}}$, of the form

$$
E_{\mathrm{RPP}}(t, \mathbf{r})=\frac{E_{0}}{N} \sum_{n,\left|k_{\perp}\right|<k_{m}}^{N} \cos \left(k_{0} x-\omega_{0} t+\mathbf{k}_{\perp}(n) \cdot \mathbf{r}_{\perp}+\Phi_{\mathbf{k}_{\perp}}\right)
$$


where $N$ is the number of diffracting elements and the phases $\Phi_{\mathbf{k}_{\perp}}$ are independent random variables taking the values 0 or $\pi$ with equal probability. For simplicity, we will assume a square phase plate that verifies $k_{\perp}(n)=2 n k_{m} / N$ and $n$ an integer with $n \in \llbracket-N / 2, N / 2 \rrbracket$ and $k_{m}=k_{0} /\left(2 f_{\sharp}\right)$. Under these conditions, and for $\langle w\rangle$ representing the statistical average of the random variable $w$, we remind that,

$$
\left\langle e^{i \Phi_{k_{1}}+i \Phi_{k_{2}}}\right\rangle=\delta\left(k_{1}-k_{2}\right) .
$$

Following the procedure introduced in Sec. II, the RPP electric field in Fourier space is

$$
\mathrm{FT}_{\omega, \mathbf{k}}\left[E_{0}(t, \mathbf{r})\right]=\frac{E_{0}}{2 N} \sum_{k_{\perp}}\left[e^{i \Phi_{k_{\perp}}} \delta\left(\omega-\omega_{0}, \mathbf{k}-\mathbf{k}_{\perp}\right)+e^{\left.-i \Phi_{k_{\perp}} \delta\left(\omega+\omega_{0}, \mathbf{k}+\mathbf{k}_{\perp}\right)\right]}\right.
$$

where $\mathbf{k}_{\perp}=k_{0} \hat{\mathbf{x}}+k_{\perp} \hat{\mathbf{y}}$ and the sum runs over $k_{\perp}$ for $\left|k_{\perp}\right|<k_{m}$. Combined with the perturbed Maxwell equations [Eq. (1)], we obtain

$$
\left(\omega_{d}^{2}-\omega_{p e}^{2}-\mathbf{k}_{d}^{2} c^{2}\right) \delta E\left(\omega_{d}, \mathbf{k}_{d}\right)=\frac{\omega_{0}^{2}}{2 N} E_{0} \sum_{k_{\perp}}\left[e^{i \Phi_{k_{\perp}}} \frac{\delta n_{e}}{n_{c}}\left(\omega_{d}-\omega_{0}, \mathbf{k}_{d}-\mathbf{k}_{\perp}\right)+e^{-i \Phi_{k_{\perp}}} \frac{\delta n_{e}}{n_{c}}\left(\omega_{d}+\omega_{0}, \mathbf{k}_{d}+\mathbf{k}_{\perp}\right)\right]
$$

Likewise, the plasma linear response, either kinetic or fluid, involves a convolution product between $E_{0}\left(\omega_{0}, \mathbf{k}_{\perp}\right)$ and $\delta E\left(\omega_{d}, \mathbf{k}_{d}\right)$ which yields,

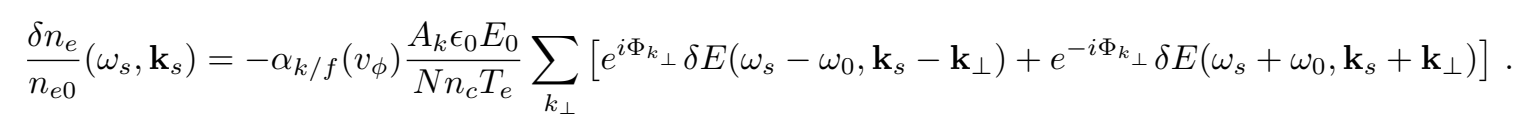

When plugging Eq. (21) into (22), two sums appear, noted with two independent indices, $k_{1}$ and $k_{2}$. As for the calculation of Eq. (12), we neglect the terms in $\delta n_{e}\left(\omega_{s} \pm 2 \omega_{0}\right)$ that are considered too far from resonance. Introducing $D_{ \pm}\left(k_{1}\right)=\left(\omega_{s} \pm \omega_{0}\right)^{2}-\omega_{p e}^{2}-\left(k_{s x} \pm k_{0}\right)^{2} c^{2}-\left(k_{s y} \pm k_{1}\right)^{2} c^{2}$ and $\mathbf{k}_{1,2}=k_{0} \hat{\mathbf{x}}+k_{1,2} \hat{\mathbf{y}}$, we obtain

$$
\frac{\delta n_{e}}{n_{e 0}}\left(\omega_{s}, \mathbf{k}_{s}\right)=-\alpha_{k / f}\left(v_{\phi}\right) A_{k} \frac{\delta n_{0}}{n_{c}} \frac{\omega_{0}^{2}}{N^{2}} \sum_{k_{1}} \sum_{k_{2}}\left[\frac{e^{i \Phi_{k_{1}}-i \Phi_{k_{2}}}}{D_{-}\left(k_{1}\right)} \frac{\delta n_{e}}{n_{e 0}}\left(\omega_{s}, \mathbf{k}_{s}-\mathbf{k}_{1}+\mathbf{k}_{2}\right)+\frac{e^{-i \Phi_{k_{1}}+i \Phi_{k_{2}}}}{D_{+}\left(k_{1}\right)} \frac{\delta n_{e}}{n_{e 0}}\left(\omega_{s}, \mathbf{k}_{s}+\mathbf{k}_{1}-\mathbf{k}_{2}\right)\right]
$$

In order to finalize the RPP dispersion relation, we will proceed to a statistical averaged using Eq. (19). On the right-hand-side, $\left\langle\exp \left(-i \Phi_{k_{1}}+i \Phi_{k_{2}}\right) \delta n_{e}\right\rangle$ can be recast as

$$
\left\langle\exp \left(-i \Phi_{k_{1}}+i \Phi_{k_{2}}\right) \delta n_{e}\right\rangle=\left\langle\exp \left(-i \Phi_{k_{1}}+i \Phi_{k_{2}}\right)\right\rangle\left\langle\delta n_{e}\right\rangle+\mathcal{C}
$$

where $\mathcal{C}$ is the correlations between $\exp \left(-i \Phi_{k_{1}}+i \Phi_{k_{2}}\right)$ and $\delta n_{e}$. The combination of Eq. (23) with (24) shows that $\mathcal{C} \propto \delta n_{0} / n_{c}$ and therefore, to leading order in $\delta n_{0} / n_{c}, \mathcal{C}$ may be neglected when averaging Eq. (23), giving

$$
1=-\alpha_{k / f}\left(v_{\phi}\right) A_{k} \frac{\delta n_{0}}{n_{c}} \frac{\omega_{0}^{2}}{N} \sum_{k_{1}}\left[\frac{1}{D_{-}\left(k_{1}\right)}+\frac{1}{D_{+}\left(k_{1}\right)}\right]
$$

Provided the phase plate has a sufficient number of elements (i.e. $N$ is large enough), we shall replace the discrete sum by a continuous one. To leading order in $\omega_{s} \ll \omega_{0}$, and making use of the pump wave dispersion relation, $\omega_{0}^{2}=\omega_{p e}^{2}+k_{0}^{2} c^{2}+k_{1}^{2} c^{2}$,

$$
D_{ \pm}\left(k_{1}\right) \simeq-\mathbf{k}_{s}^{2} c^{2} \pm 2\left(\omega_{s} \omega_{0}-k_{s x} k_{0} c^{2}-k_{s y} k_{1} c^{2}\right),
$$

so that,

$$
\begin{array}{r}
\frac{1}{N} \sum_{k_{1}} \frac{1}{D_{ \pm}\left(k_{1}\right)} \simeq \frac{1}{2 k_{m}} \mathrm{P} . \mathrm{V} \cdot \int_{-k_{m}}^{k_{m}} \frac{d k_{1}}{D_{ \pm}\left(k_{1}\right)} \\
\simeq \frac{\mp 1}{4 k_{m} k_{s y} c^{2}} \ln \left[\frac{-\mathbf{k}_{s}^{2} c^{2} \pm 2\left(\omega_{s} \omega_{0}-k_{s x} k_{0} c^{2}-k_{s y} k_{m} c^{2}\right)}{-\mathbf{k}_{s}^{2} c^{2} \pm 2\left(\omega_{s} \omega_{0}-k_{s x} k_{0} c^{2}+k_{s y} k_{m} c^{2}\right)}\right] .
\end{array}
$$

We introduced, P.V. $\int f$, the principal value of $\int f$. The combination with Eq. (25) yields

$$
\begin{aligned}
& \frac{-4 k_{m} k_{s y} c^{2}}{\alpha_{k / f}\left(v_{\phi}\right) A_{k} \omega_{0}^{2} \delta n_{0} / n_{c}} \\
& \simeq \ln \left[\frac{\left(\mathbf{k}_{s}^{2} c^{2}-2 k_{s y} k_{m} c^{2}\right)^{2}-4\left(\omega_{s} \omega_{0}-k_{s x} k_{0} c^{2}\right)^{2}}{\left(\mathbf{k}_{s}^{2} c^{2}+2 k_{s y} k_{m} c^{2}\right)^{2}-4\left(\omega_{s} \omega_{0}-k_{s x} k_{0} c^{2}\right)^{2}}\right] .
\end{aligned}
$$

In order to avoid the branch cuts of the complex logarithm, it is convenient to recast the dispersion relation 
(a) Kinetic, $\log _{10}\left(\Gamma / k_{0}\right)$

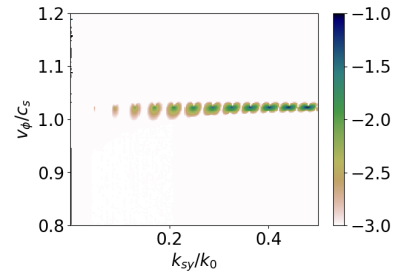

(c) Fluid, $\log _{10}\left(\Gamma / k_{0}\right)$

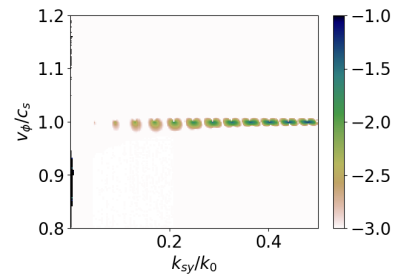

(b) Kinetic, $\Re\left(k_{s x} / k_{0}\right)$

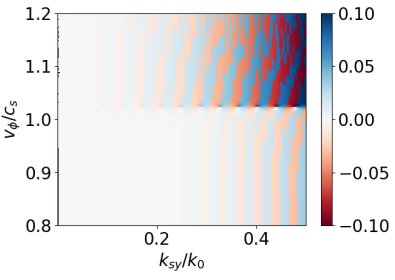

(d) Fluid, $\Re\left(k_{s x} / k_{0}\right)$

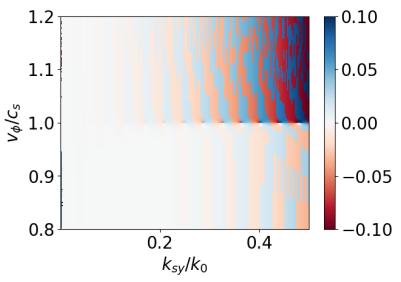

Figure 3. Kinetic (a,b) and fluid (c,d) resolution of Eqs. (29)(31) for the same parameters than in Fig. 1 assuming a RPP beam given by Eq. (18) with $f_{\sharp}=8$. The thermal correction of Eq. (5) verifies $A_{k} \leq 0.65$ for $k_{s y} \geq 0.1 k_{0}$.

into

$$
u^{2}-2 \frac{v_{\phi}}{\eta c} u+\frac{v_{\phi}^{2}}{\eta^{2} c^{2}}-\frac{1}{4} A=0,
$$

$A=\frac{1}{1-B}\left[\left(\frac{k_{s}^{2}}{\left|k_{s}\right| k_{0}}-\frac{k_{s y}}{\left|k_{s}\right| f_{\sharp}}\right)^{2}-\left(\frac{k_{s}^{2}}{\left|k_{s}\right| k_{0}}+\frac{k_{s y}}{\left|k_{s}\right| f_{\sharp}}\right)^{2} B\right]$,

$$
B=\exp \left(\frac{-4 k_{m} k_{s y} c^{2}}{\alpha_{k / f}\left(v_{\phi}\right) A_{k} \omega_{0}^{2} \delta n_{0} / n_{c}}\right) \text {. }
$$

Note that the dependence of $A$ on $k_{s x}$ may be neglected (provided $\left|k_{s x}\right| \ll\left|\mathbf{k}_{s}\right|$ ) so that $u=k_{s x} /\left|\mathbf{k}_{s}\right|$ may verify a second order polynomial equation given by Eq. with

$$
\begin{array}{r}
A \simeq \frac{1}{1-B}\left[\left(\frac{\left|k_{s}\right|}{k_{0}}-\frac{1}{f_{\sharp}}\right)^{2}-\left(\frac{\left|k_{s}\right|}{k_{0}}+\frac{1}{f_{\sharp}}\right)^{2} B\right], \\
B \simeq \exp \left(\frac{-4 k_{m}\left|k_{s}\right| c^{2}}{\alpha_{k / f}\left(v_{\phi}\right) A_{k} \omega_{0}^{2} \delta n_{0} / n_{c}}\right) .
\end{array}
$$

The only difference between the plane wave and the RPP dispersion relations holds in the last term of the 1.h.s. of Eqs. (17) and (29). As expected, the latter coincide when a Taylor expansion of $A$ [Eq. (32)] to first order in $1 / f_{\sharp}$ is done, i.e. for a vanishing RPP beam spectral width, $2 k_{m}=k_{0} / f_{\sharp}$. Care has been taken to verify that the RPP dispersion relations with $f_{\sharp} \geq 50$ yield very similar results than Eq. (17). We also verified that extracting $k_{s x}$ from a numerical resolution of Eq. (25) also yields similar results for 100 phase plate elements (i.e. when

(a) Kinetic, $\log _{10}\left(\Gamma / k_{0}\right)$

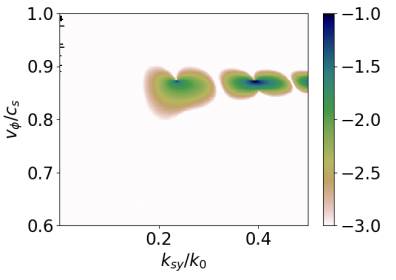

(c) Fluid, $\log _{10}\left(\Gamma / k_{0}\right)$

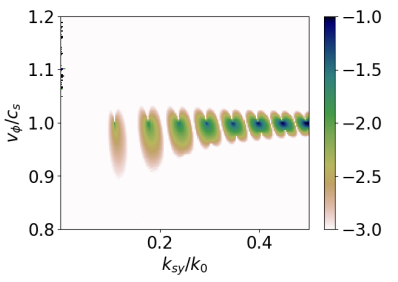

(b) Kinetic, $\Re\left(k_{s x} / k_{0}\right)$

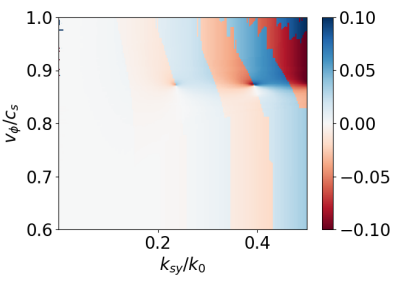

(d) Fluid, $\Re\left(k_{s x} / k_{0}\right)$

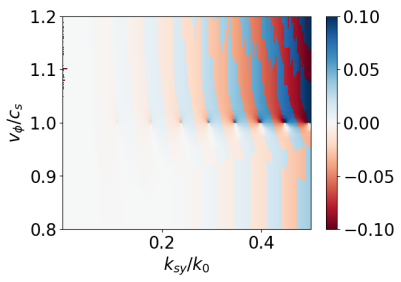

(e) lineout at $v_{\phi}=0$

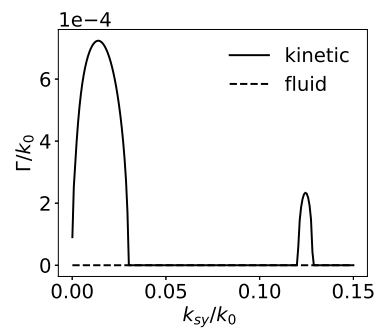

Figure 4. Kinetic (a,b) and fluid (c,d) resolution of Eqs. (29)(31) for the same parameters than in Fig. 2 assuming a RPP beam given by Eq. (18) with $f_{\sharp}=8$. The value of the non-local correction [Eq. (5)], is obtained with the carbon parameters, corresponding to the smallest electron-ion meanfree-path and verifies $A_{k} \leq 1.4$ for $k_{s y} \geq 0.1 k_{0}$. Use is made of the mean charge number and mean mass number for calculating the sound speed and the normalized Landau damping rate $c_{s}$ and $\gamma_{0}$. Panel (e) corresponds to lineouts at $v_{\phi}=0$ of (a) and (c).

the sum runs over $N=100$ regularly spaced elements of $\left.\left[-k_{m}, k_{m}\right]\right)$.

The solutions of the RPP dispersion equations (29)(31) stem from a non linear algebraic solver, or using a dichotomous algorithm applied at fixed $v_{\phi}$ and starting from low values of $\left|\mathbf{k}_{s}\right|$. The initial guess is seeded by the approximated solutions of Eqs. (29), (32) and (33), yielding convergence toward the exact solution. Moreover, if $k_{x}\left(\omega_{s}, k_{y}\right)$ is a solution of Eq. (28), the symmetry properties of our free-drift velocity dispersion relations imply that $-k_{x}\left(-\omega_{s}, k_{y}\right)$ and $k_{x}^{\star}\left(-\omega_{s}, k_{y}\right)$ are also solutions (where $z^{\star}$ is the complex conjugate of $z$ ), therefore helping to constrain the numerical solver to converge toward a single eigenmode. The spatial growth rate and wavevector longitudinal component are illustrated in Figs. 3 and 4 with identical plasma and laser parameters than in Figs. 1 and 2, respectively and with $f_{\sharp}=8$. Finally, the assumption made in deriving Eqs. (32)-(33) $\left(\left|k_{s x}\right| \ll\left|\mathbf{k}_{s}\right|\right)$ seems, for the parameters of in- 
terest here, scarcely verified (see Figs. 3 and 4 where for $k_{s y} / k_{0}=0.4,\left|k_{s x}\right| / k_{0}$ reaches $\left.\sim 0.1\right)$.

Hence, regarding the FSBS, no simple stability criterion could be extracted from our dispersion relation such as the one proposed in Refs. [13, 36, 38]. Figures 3(b,d) and $4(\mathrm{~b}, \mathrm{~d})$ also suggest that, in contrast with the plane wave case, the FSBS growing of a RPP beam induces acoustic fluctuations which propagating direction may have a finite $x$ component.

\section{B. Filamentation of a spatially smoothed beam}

Regarding the $\mathrm{H}^{+}$-plasma case (Fig. 3), the propagation of the smoothed beam is, as expected [9, 14, 51], stable regarding the filamentation instability, i.e. no growth is obtained at $v_{\phi}=0$. In that case, Eq. (29) simplifies to $u^{2}=A / 4$ which can be recast into a second order polynomial equation in $k_{s x}^{2}$,

$$
\begin{array}{r}
k_{s x}^{4}+z_{2} k_{s x}^{2}+z_{0}=0, \\
z_{2}=2 k_{0}^{2}\left(\frac{k_{-}-k_{+} B}{k_{0}(1-B)}-2\right), \\
z_{0}=k_{0}^{2} \frac{k_{-}^{2}-k_{+}^{2} B}{(1-B)}, \\
k_{ \pm}=\frac{k_{s y}^{2}}{k_{0}} \pm \frac{k_{s y}}{f_{\sharp}} .
\end{array}
$$

The unstable solution of the above equation is purely imaginary and corresponds to the spatial growth rate of a spatially smoothed laser filamentation instability. Physically, the speckle typical size, $f_{\sharp} \lambda_{0}$, remains smaller than the most unstable filamentation wavelength $[\sim 10 \mu \mathrm{m}$ in Fig. 1(a)] thus preventing the transverse standing electrostatic wave to grow. The artificial increase of $f_{\sharp}$ in excess of $\sim 25$, i.e. yields a finite value of the filamentation spatial growth rate as the speckle size starts to be comparable with the plane wave most unstable wavelength. For more moderate $f_{\sharp}$-numbers, the propagation of a laser may also remains filamentation-unstable, despite the use of random phase plates, in the case of a multi-ion species plasmas such as in Fig. 4. Although less filamentation unstable than in the plane wave case [see Figs. 2(a,c) and 4(e)], the RPP beam has a finite kinetic growth rate maximized at $\Gamma \sim 7 \times 10^{-4} k_{0}$ and $\left|k_{s}\right| \sim 10^{-2} k_{0}$. Hence, the estimated gain of $\sim 12$ for a millimeter of propagation (with a wavelength of $\sim 35 \mu \mathrm{m}$ ) indicates that keV-range plasmas may produce significant RPP-laser filamentation, at least in the multi-ion species case. Notably, another filamentation mode exists around the speckle size $\left|k_{s}\right|=2 k_{0} /\left(2 f_{\sharp}\right)=0.125 k_{0}$, albeit with a smaller growth rate $\Gamma \sim 2 \times 10^{-4} k_{0}$.

Unfortunately, the use of random phase plates seems to completely stabilize the filamentation in the fluid framework [see dashed line, Fig. 4(e)], demonstrating that hydrodynamic codes fail to capture correctly the laser filamentation in a multi-ion species plasma. Additionally, (a) Setup
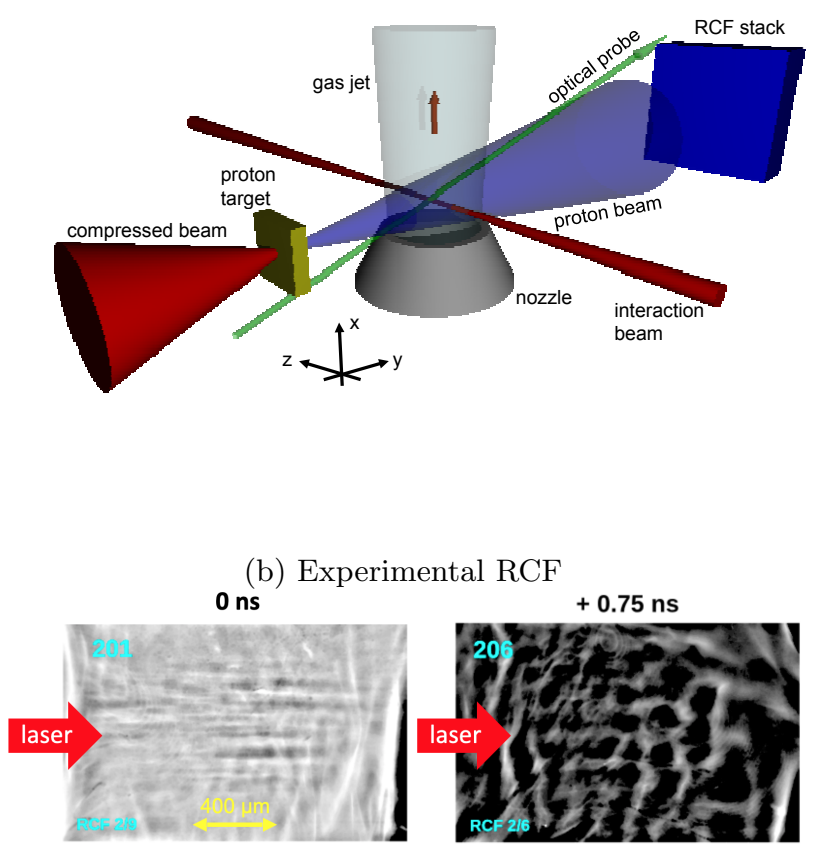

Figure 5. (a) Sketch of the experimental setup. (b) Experimental RCF (obtained using $3 \mathrm{MeV}$ protons) from two shots of the LULI experiment, at 0 and $0.75 \mathrm{~ns}$ after the maximum intensity reaches the center of the plasma slab. The scale indicated at the bottom of the left image applies to both and refers to the target plane.

the kinetic instability characterized in Fig. 4(e) is mainly thermal as it is completely stabilized when replacing $A_{k}$ in Eq. (34) by its collisionless value, 1/2.

\section{Comparison with proton radiographs of a RPP pulse propagating in a helium gaz jet}

A low temperature plasma may also be filamentationunstable to the propagation of a RPP pulse. For a single ion species plasma, and provided $Z_{i} T_{e} \gg T_{i}$, we recall that $\alpha_{f}(0)=1 \simeq \alpha_{k}(0)$, for this reason, the filamentation growth coincides in both kinetic and hydrodynamic frameworks. Hence, restricting, in this section, the analysis to the fluid plasma response, we aim at comparing our predictions with the laser filamentation observed experimentally at relatively low temperature.

The experiment, detailed in Ref. [14], uses a tightly focused $\left(f_{\sharp}=3\right)$ RPP pulse, therefore probably out of reach of our dispersion relations where we neglected diffraction on the pump wave envelope [in Eq. (18)]. Hence the choice has thus been made to lean on a longer focus experiment performed using the LULI 100TW laser 
(a) $T_{e}(t)$ and $I_{0}(t)$

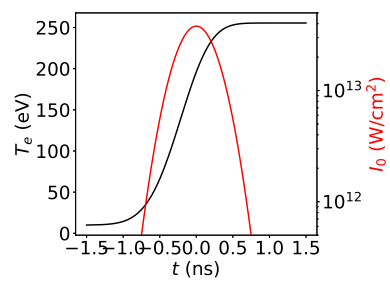

(b) $\Gamma \times L$

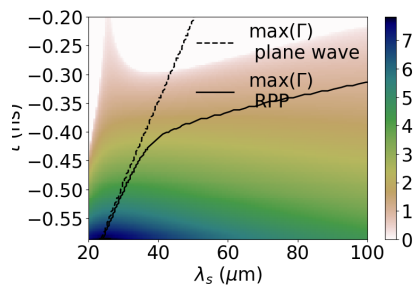

film corresponding to a range, and thus a particular proton energy (determined using the code SRIM [61]). The spatial resolution of the proton radiography is given by the virtual source size which is $\sim 5$ microns [62] and the temporal resolution is given by the time required by the protons to cross the interaction zone, i.e. $\sim 4 \mathrm{ps}$ for 3 $\mathrm{MeV}$ protons. The different times shown in Fig. 5(b) correspond to different shots.

Figure 6. (a) Temporal evolution of the electron temperature from the resolution of $(3 / 2) n_{e} d_{t} T_{e}=\nu_{B} I_{0} 2^{-t^{2} / \tau^{2}} / c$ with $\tau=300 \mathrm{ps}$ and $T_{e}(-1.5 \mathrm{~ns})=10 \mathrm{eV}$ (left axis, black). The intensity evolution is superimposed as a red line (right axis). (b) Filamentation growth rate corresponding to the unstable solution of Eq. (34) normalized to the plasma length $L$ for $v_{\phi}=0$ and in the fluid framework [Eq. (10)] with $I_{0}=3.8 \times 10^{13} \mathrm{~W} / \mathrm{cm}^{2}, 2 \pi / k_{0}=1 \mu \mathrm{m}, f_{\sharp}=24, Z_{i}=2$, $A=4, n_{e}=10^{19} \mathrm{~cm}^{-3}, L=1 \mathrm{~mm}$ and $Z_{i} T_{e} / T_{i}=5$. The growth rate maximum is superimposed as a black line in the $\mathrm{RPP}$ case and as a dashed line in the plane wave case [Eq. (17)].

facility at the fundamental wavelength of 1.053 micron. The beam after amplification is split in several beams: a vacuum compressed beam (compressed beam in Fig. 5 (a), with a duration of $350 \mathrm{fs}$ ) used for the production of protons as a diagnostic [58], and an interaction beam (interaction beam in Fig. 5(a), uncompressed, $\tau=300$ ps HWHM). The interaction beam is linearly polarized in the plane $(y, z)$. As shown in Fig. 5(a), both beams are focused at $90^{\circ}$. An optical time slide on one beam allowed to set a variable delay between the compressed and the interaction beam with sub-ps precision. The compressed beam was focused, using an $f / 24\left(f=2.1 \mathrm{~m}, f_{\sharp}=24\right)$ lens coupled to a RPP, producing an intensity at focus of $I_{0}=3.8 \times 10^{13} \mathrm{~W} / \mathrm{cm}^{2}$, onto a $L=1 \mathrm{~mm}$ diameter supersonic Helium gas jet corresponding to $n_{e} / n_{c} \simeq 10^{-2}$ for a fully ionized plasma. Proton radiography of the interaction was performed using a laminar beam of protons generated by the compressed beam. To this end, the latter was focused (with a FWHM of $\sim 6$ microns) to a peak intensity of $4 \times 10^{19} \mathrm{~W} / \mathrm{cm}^{2}$ on 10 microns thick $\mathrm{Au}$ foils positioned at $d=3.5 \mathrm{~mm}$ away from the center of the gas jet. This produced [59] a beam of laminar protons having a broad energy spectrum extending here from 0 to $15 \mathrm{MeV}$. The protons originate from hydrogenated contaminants on the target surface. They were detected downstream, at $D=43.5 \mathrm{~mm}$ from the gas jet, by a stack of radiochromic films (RCFs) [60] protected by a $14 \mu \mathrm{m}$-thick Al range filter. The resulting magnification of the proton projection onto the RCFs was $M=(d+D) / d=13.1$. RCFs are preferentially sensitive to penetrating protons, which have a large specific energy-loss and produce a high contrast image. Since we used a stack of films, and since protons have a welldefined range in matter, that stack arrangement therefore allowed a coarse resolution in proton energy, each

An estimate of the plasma temperature evolution may be obtained easily in this low temperature and low density experiment $\left(T_{e} \lesssim 250 \mathrm{eV}, n_{e} / n_{c}=10^{-2}\right)$ by neglecting the electron thermal diffusion and accounting only for the inverse Bremsstrahlung laser absorption calculated on the transversely averaged intensity (neglecting the speckle-scale intensity fluctuations). The resulting electron temperature evolution, $(3 / 2) n_{e} d_{t} T_{e}=\nu_{B} I_{0} 2^{-t^{2} / \tau^{2}} / c\left(\right.$ where $\nu_{B} \propto T_{e}^{-3 / 2}$ is the bremsstrahlung laser absorption coefficient) has been resolved and is illustrated in Fig. 6(a), showing that $T_{e} \lesssim$ $150 \mathrm{eV}$ is obtained before the pulse maximum intensity, i.e. for $I \lesssim 3 \times 10^{13} \mathrm{~W} / \mathrm{cm}^{2}$. The colormap of Fig. $6(\mathrm{~b})$ illustrates the RPP filamentation spatial growth normalized to the plasma length as a function of wavelength and time, for the experimental parameters, intensity and electron temperature evolution discussed above [see Fig. 6(a)]. Moreover, the RCF signal late-time evolution suggests a ratio $Z_{i} T_{e} / T_{i} \simeq 4.5$ as discussed in appendix. Hence, the RPP growth rate maximum (black plain line) demonstrates that a reasonable gain is obtained, $\Gamma L \gtrsim 3$, when $t \simeq-0.35 \mathrm{~ns}, I \sim 10^{13} \mathrm{~W} / \mathrm{cm}^{2}$ and $T_{e} \lesssim 80 \mathrm{eV}$. As soon as $t>-0.2 \mathrm{~ns}$, the density fluctuations should cease growing as the gain drops below unity and subsequently be damped by the Landau process over a few $\left(\gamma_{0} c_{s} 2 \pi / \lambda_{s}\right)^{-1} \sim 1 \mathrm{~ns}$. This suggests that the filamentation grows and saturates rapidly before the most energetic part of the beam reaches the center of the gaz jet, leading to density fluctuations of wavelength $\lambda_{F} \sim 60 \mu \mathrm{m}$ and measurable for $t>0 \mathrm{~ns}$. The resulting electrostatic field is able to deflect the probing protons causing the proton dose modulation illustrated in Fig. 5(b). The estimated experimental wavelength of $\simeq 77 \mu \mathrm{m}$, obtained by maximizing the Fourier transformed experimental signal over a central lineout, fairly agrees with our RPP dispersion relations (see Fig. A1(a) in appendix). Note that the textbook plane wave filamentation dispersion relations [Eq. (17) for $v_{\phi}=0$ ] predict a smaller dominant wavelength of $\lambda_{F} \lesssim 35 \mu \mathrm{m}$ (when $T_{e} \lesssim 80 \mathrm{eV}$ ), illustrated as a dashed black line in Fig. 6(b). A large disagreement of the RPP and plane wave most unstable wavelengths is obtained when $\lambda_{s}>f_{\sharp} \lambda_{0}=24 \mu \mathrm{m}$ [in Fig. 6(b), as discussed in Sec. III B], demonstrating the significance of the random phase plate dispersion relations regarding realistic conditions [63]. 


\section{Forward Brillouin scattering of a spatially smoothed beam}

Figures 3 and 4 show also significant differences compared to the propagation of a plane wave regarding the forward Brillouin scattering. Either fluid or kinetic, for single or multiple ion species, the spatial growth rate appears much more peaked around $v_{\phi} \simeq c_{s}$ [or $v_{\phi} \simeq 0.8 c_{s}$ for the kinetic $\mathrm{CH}$ case, Fig. 4(a)] and the propagation of the RPP pulse more unstable $\left[\Gamma / k_{0} \sim 0.1\right.$, Fig. $3(\mathrm{a})$ and Fig. $4(\mathrm{a})]$ than for a plane wave case $\left[\Gamma / k_{0} \simeq 2 \times 10^{-4}\right.$, Fig. 1(a)]. Therefore degrading the pump spatial coherence decreases or potentially suppresses the filamentation instability, however, at the expense of favoring the spatial growth of the forward Brillouin instability. The forward scattering of a RPP beam ensues from the superposition of all the pulse spectral contributions, the final superposition of acoustic waves may be constructive or destructive depending on the wavevector direction and amplitude relative to the plasma resonance [characterized by $\alpha_{k / f}$, Eqs. (9)-(10)]. As a consequence, the spatial growth consists in a succession of peaks aligned around $v_{\phi} \simeq c_{s}$ and spread from $\left|k_{s}\right|=0$ to a fraction of $k_{0}$. The separation of these peaks [a few $10^{-2} k_{0}$ for Fig. $3(\mathrm{a})$ and $\sim 10^{-1} k_{0}$ for $4(\mathrm{a})$ ], depends on the propagation properties of the driven acoustic waves and the plasma response such as the width of the resonance. Figures 3(a,c) and $3(\mathrm{~b}, \mathrm{~d})$ are very similar demonstrating that, even for moderate values of $Z_{i} T_{e} / T_{i} \geq 3$, the fluid framework satisfactorily captures the spatial growth of the FSBS in the single ion species case and that using the non-local correction of Eq. (5) is justified regarding the kinetic results provided we may neglect the Coulomb collisions contribution to the acoustic wave damping. In the multi-ion species case however [see Figs. 4(a,c)], twice more unstable peaks are evidenced in the fluid (c) than in the kinetic (a) framework, whereas both approaches exhibit similar growth rate maximums.

The quality of the RPP beam propagation can be estimated by comparing the spectral width of the growth rate with the beam aperture in vacuum $k_{m} / k_{0}=$ $1 /\left(2 f_{\sharp}\right) \simeq 0.062$. For the $\mathrm{H}^{+}$-plasma, the range $0.05 \lesssim$ $k / k_{0} \lesssim 0.4$ is unstable thus leading to the increase of the $f_{\sharp}$-cone angle from $1 /\left(2 f_{\sharp}\right)=3.6^{\circ}$ to $\sim 21^{\circ}$. Hence, a substantial modification of the beam properties could appear during its propagation thus affecting the energy deposition. Regarding the CH-plasma, one may notice that unlike for the single ion case, the peaks are located around $v_{\phi}=0.8 c_{s}$, implying a lower acoustic frequency (than for the single ion species case or than the fluid calculations) and therefore less red-shifting of the scattered wave.

The two and six unstable peaks shown in Figs. 4(a) and (c) demonstrate that the density fluctuation should present discrete growing modes corresponding to $\sim$ $\left(16^{\circ}, 21^{\circ}\right)$ and $\sim\left(8^{\circ}, 11^{\circ}, 14^{\circ}, 17^{\circ}, 19^{\circ}, 21^{\circ}\right)$ light scattering angles, respectively. Although a similar maximum growth rate is predicted by the kinetic and fluid calcu- lations, different scattering directions are obtained. The fluid framework suffers, in some cases, from an ill-forecast of the scatter spectral properties.

\section{COMPARISON WITH HYDRODYNAMIC SIMULATION}

In this section we aim at validating the derived fluid spatial growth rate through a comparison with hydrodynamic simulations. Regarding the kinetic counterpart, the corresponding full "particle-in-cell" simulations remain out of reach of present super-computers and are therefore out of the scope of the present manuscript.

We thus performed two HERA hydrodynamic simulations with a paraxial resolution of the RPP beam propagation [30]. A 2D domain of size $L_{x} \times L_{y}=2000 \times$ $512 \mu \mathrm{m}^{2}$ is used. A RPP beam propagating in the $x$ direction is injected at the left boundary, $x_{\mathrm{BC}}=0$, with a $\lambda_{0}=0.35 \mu \mathrm{m}$-wavelength and an averaged intensity of $I_{0}=6 \times 10^{14} \mathrm{~W} / \mathrm{cm}^{2}$. The focal spot is located at the center of the simulation domain, $x_{\mathrm{foc}}=1000 \mu \mathrm{m}$ with a focal number of $f_{\sharp}=8$, and a spatial and temporal envelope following

$$
\begin{aligned}
\hat{g}(y) & =\exp \left(-|y|^{o} / 2 \sigma_{g}^{o}\right), \\
h(t) & =\min \left(t / \tau_{h}, 1\right),
\end{aligned}
$$

respectively with $\tau_{h}=1 \mathrm{ps}, o=5$ and $\sigma_{g}=200 \mu \mathrm{m}$. For sake of simplicity and comparison purposes, the bremsstrahlung energy deposition is neglected and the non-local thermal correction of Eq. (5) is accounted for. Moreover a barotropic gas is assumed with an electron density of $n_{e}=0.1 n_{c}$ and outflow boundary for the fluid. The mesh size is $d x=0.325 \mu \mathrm{m}, d y=0.0625 \mu \mathrm{m}$ with a Landau acoustic damping rate calculated on Eq. (6) with the initial plasma parameters. The acoustic Landau damping operator is computed transversely to the laser direction in the Fourier space, as introduced in Ref. [64], described in [63] and used in [65-67]. In order to focus on the FSBS, we do not account for any back-scattering in our simulations.

As illustrated in Figs. $7(\mathrm{a}, \mathrm{d})$, the RPP beam presents a cone angle increase as soon as $x \gtrsim 500 \mu \mathrm{m}$ at $t=100 \mathrm{ps}$ (and later on). Attributed to the FSBS, its growth may be characterized and compared to our predictions.

The density fluctuation spectrum [Fig. 7(b) for the case $\left.T_{i}=300 \mathrm{eV}\right]$ is peaked along the acoustic mode ( $\omega=k_{y} c_{s}$ as a black solid line) as expected. We may extract an effective spatial growth rate from our numerical results by proceeding to the spatial (in the $y$ direction) and temporal Fourier transform of two lineouts of the density fluctuations, at $x=x_{1}$ and $x_{2}$ and following,

$$
\Gamma=\frac{1}{2\left(x_{2}-x_{1}\right)} \ln \left[\frac{\left|\delta n_{e}\left(x_{2}, k_{y}, \omega\right)\right|^{2}}{\left|\delta n_{e}\left(x_{1}, k_{y}, \omega\right)\right|^{2}}\right],
$$

over a time range during which the system does not evolve much. For the parameters addressed here, the 
(a) $\log _{10}\left(I\left[\mathrm{~W} \cdot \mathrm{cm}^{-2}\right]\right)$

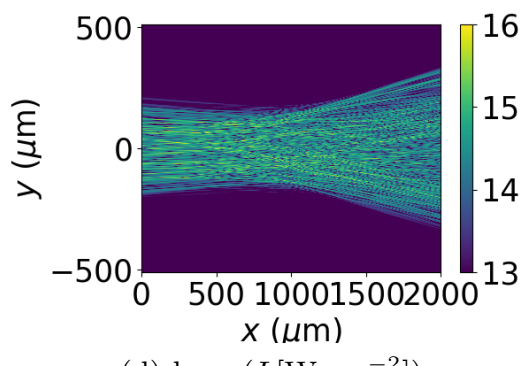

(d) $\log _{10}\left(I\left[\mathrm{~W} . \mathrm{cm}^{-2}\right]\right)$

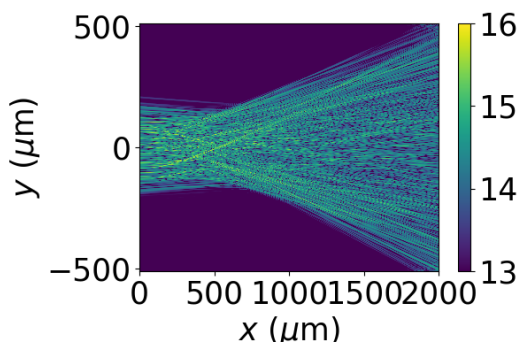

(b) $\log _{10}\left(\left|\delta n_{e}\left(\omega, k_{y}, x_{2}\right) / n_{c}\right|^{2}\right)$

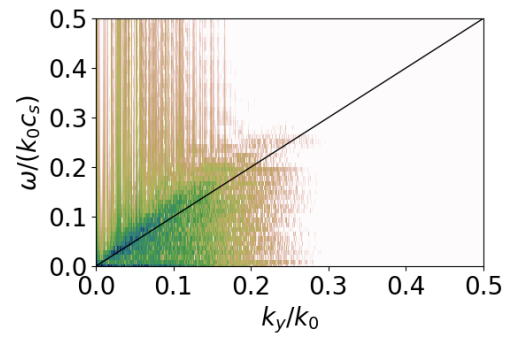

(e) Theory, $\log _{10}\left(\Gamma / k_{0}\right)$

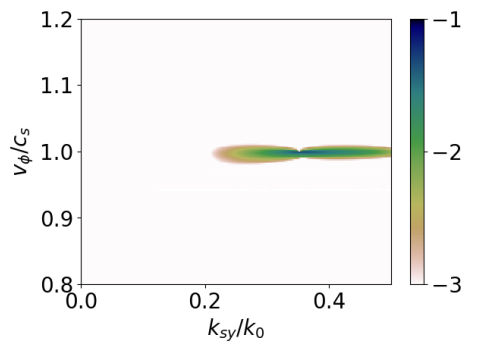

(c) $\Gamma / k_{0}$

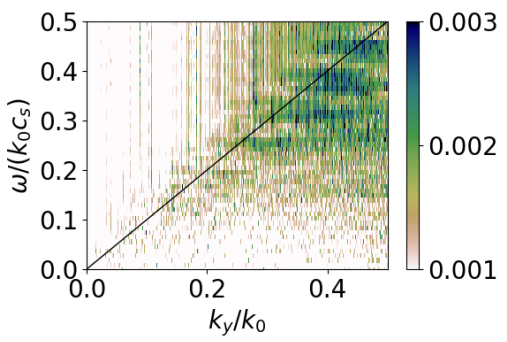

(f) $\Gamma / k_{0}$

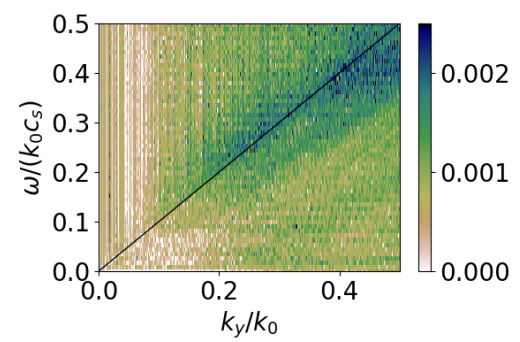

Figure 7. Simulation results for the $\mathrm{H}^{+}$plasma with $n_{e}=0.1 n_{c}, T_{e}=1 \mathrm{keV}$ and $T_{i}=300 \mathrm{eV}(\mathrm{a}, \mathrm{b}, \mathrm{c})$ and $T_{i}=100 \mathrm{eV}(\mathrm{d}, \mathrm{e}, \mathrm{f})$. $(\mathrm{a}, \mathrm{d})$ Intensity profile at $t=100 \mathrm{ps}$ and (b) spatio-temporal spectrum at $x_{2}=250 \mu \mathrm{m}$ of $\delta n_{e}\left(\omega, k_{y}, x_{2}\right)$. (c,f) Effective spatial growth rate extracted from the numerical results between 25 and 135 ps (c) and between 40 and 135 ps (f) following Eq. (37) with $\left(x_{1}, x_{2}\right)=(200,300) \mu \mathrm{m}(\mathrm{c})$ and $\left(x_{1}, x_{2}\right)=(50,150) \mu \mathrm{m}(\mathrm{f})$. The corresponding theoretical growth rates as discussed in Sec. III A are illustrated in Fig. 3(c) and panel (e) for $T i=300$ and $100 \mathrm{eV}$, respectively. The acoustic eigenmode $\omega=k_{y} c_{s}$ is superimposed on $(\mathrm{b}, \mathrm{c}, \mathrm{f})$ as black plain lines.

laser propagation seems to reach a steady state in a few $10 \mathrm{ps}$, hence the temporal Fourier transform has been performed for $t \in[25,135] \mathrm{ps}$ and $[40,150] \mathrm{ps}$ for $T_{i}=300$ and $100 \mathrm{eV}$, respectively. Hence, Figs. 7(c,f) illustrate for both cases the effective spatial growth rate in the plane $\left(\omega, k_{y}\right)$ indicating stability around $\omega=0$, i.e. no filamentation instability, consistently with the theoretical predictions and with Figs. 7(a,d). Interestingly, the growth rate along the acoustic mode, marked by the black plain line $\omega=k_{y} c_{s}$ in Fig. 7(c), exhibits a succession of peaks for $k_{y}>0.2 k_{0}$ consistently with our theory [Fig. 3 (c)]. Their separation of $\sim 0.05 k_{0}$ correctly agrees with Fig. 3(c). Moreover, they extend from $k_{y} \sim 0.2 k_{0}$ to $0.5 k_{0}$, therefore exceeding the initial transverse spectral width of $k_{0} / 2 f_{\sharp} \simeq 0.062 k_{0}$ and explaining the large cone angle observed for $x>500 \mu \mathrm{m}$ in Fig. 7 (a) of $\sim 0.2 k_{0}$ (i.e. $11^{\circ}$ ). When $T_{i}=100 \mathrm{eV}$, the theoretical predictions [Fig. $7(\mathrm{e})$ ] yields only one unstable peak located between $\sim 0.2 k_{0}$ and $\sim 0.5 k_{0}$. Likewise, a single large cluster (between $k_{y} / k_{0} \sim 0.2$ and 0.5 ) may be recognized in Fig. $7(\mathrm{f})$ with a dominant feature around $k_{y} \sim 0.4 k_{0}$, as predicted by our dispersion relations.

Regarding the density fluctuations growth level, the $100-150 \mu \mathrm{m}$-long and $105-110 \mathrm{ps}-$ long window over which the Fourier transform has been performed for resolution purposes, does not allow a quantitative comparison with the theoretical predictions. By contrast, the scattered field growth is more suitable to quantitative comparisons. Indeed, the linearized paraxial propagation of the perturbed fields $\delta E$, when accounting for diffraction, verifies

$$
\partial_{x} \delta E\left(\mathbf{k}_{d}, t\right)+\frac{i \mathbf{k}_{d}^{2}}{2 k_{0}} \delta E\left(\mathbf{k}_{d}, t\right)=-\frac{i}{2 \pi} \frac{k_{0} n_{0}}{2 n_{c}} E_{0} \otimes \frac{\delta n_{e}}{n_{e}} .
$$

For illustration purposes, we will model the spatial growth rate spectrum by a succession of Dirac functions, so that

$$
\frac{\delta n_{e}}{n_{e}}\left(\mathbf{k}_{s}\right) \simeq \frac{\delta n_{0}}{n_{0}} \sum_{\mathbf{k}_{c}} e^{i \Gamma_{c} x} \delta\left(\mathbf{k}_{s}-\mathbf{k}_{c}\right)
$$

where $\left(\mathbf{k}_{c}, \Gamma_{c}\right)$ are the location and amplitude of the peaks. Hence, the solution of Eq. (38), using the pump fields of Eq. (20), allows to compute the perturbed fields, $\delta E$, as a function of the phase plate random variables $\Phi_{k}$. Finally, the corresponding averaged perturbed intensity [using Eq. (19)] defined as $\delta I \propto\left\langle E_{0} \otimes \delta E\right\rangle$ reads

$\delta I\left(\mathbf{k}_{d}\right) \propto \sum_{\mathbf{k}_{c}} \frac{e^{\Gamma_{c} x}-e^{-i\left(\mathbf{k}_{d}+\mathbf{k}_{c}\right)^{2} x / 8 k_{0}}}{\left(\mathbf{k}_{d}+\mathbf{k}_{c}\right)^{2}-8 i k_{0} \Gamma_{c}} \mathrm{H}\left(2 k_{m}-\left|\mathbf{k}_{d}-\mathbf{k}_{c}\right|\right)$,

where $\mathrm{H}$ is the Heaviside step function. This demonstrates that although high frequency density fluctuations may grow significantly, the $\sim \mathbf{k}_{d}^{-2}$ factor in front of the exponential due to diffraction tends to favor FSBS growth for small wavevectors. For highly unstable systems such as the one addressed here, saturation occurs 

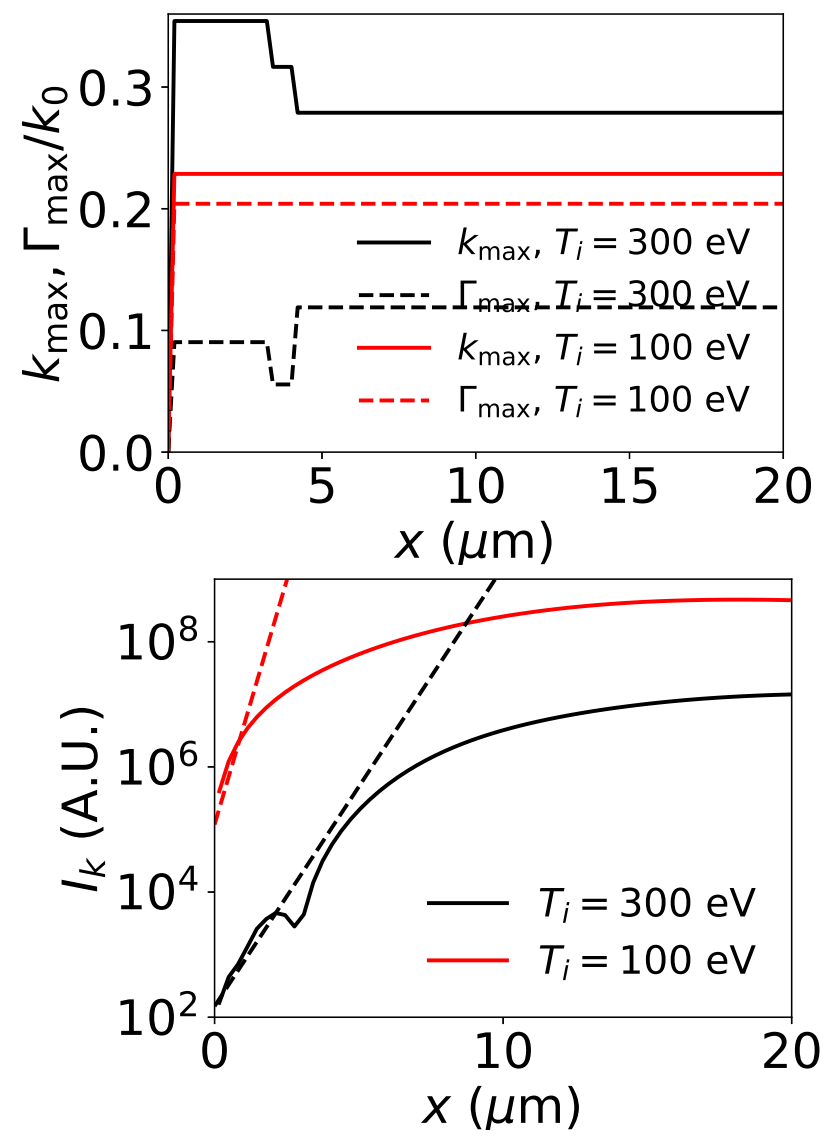

Figure 8. (top) Effective growth rate (dashed lines) and wavevector (plain lines) that maximise Eq. (40), $\left(\Gamma_{\max }, \mathbf{k}_{\max }\right)$, normalized to $k_{0}$ for both hydrogen simulations of Fig. 7. (bottom) Lineout of the intensity transverse Fourier transform at $k_{y}=0.26 k_{0}\left(T_{i}=300 \mathrm{eV}\right.$, black plain line $)$ and $k_{y}=0.22 k_{0}\left(T_{i}=100 \mathrm{eV}\right.$, red plain line $)$. The theoretical exponential growth with $\Gamma_{c}=0.09 k_{0}\left(T_{i}=300 \mathrm{eV}\right.$, black dashed line $)$ and $\Gamma_{c}=0.2 k_{0}\left(T_{i}=100 \mathrm{eV}\right.$, red dashed line $)$ are superimposed.

rapidly. Hence, when the pump depletion is reached, the wavevector that saturates first is the one that maximises $\delta I$ and does not necessarily coincide with the fluctuation density growth rate maximum. In these conditions, the field effective growth rate and wavevector, (i.e. the effective scattered field f-cone angle), may be related to the wavevector that maximises Eq. (40) and the corresponding growth rate peak amplitude and location, $\left(\Gamma_{\max }, \mathbf{k}_{\max }\right)$.

Figure 8(a) illustrates the spatial evolution of $\left(\Gamma_{\max }, \mathbf{k}_{\max }\right)$ for the two simulations of Fig. 7 and demonstrates that, the wavevector and peak growth rate that maximize $\delta I$ remain essentially unchanged over the first 20 microns of the laser propagation (and corresponding to a $>10^{10}$-factor increase of $\left.\delta I\right)$. The exponential growths of $\delta I\left(k_{y}=k_{\max }\right)$ extracted from the hydrodynamic simulations and illustrated in Fig. 8(b) as plain lines, correctly agree with our theoretical predictions (as dashed lines) and show that saturation is reached after $\sim 10 \mu \mathrm{m}$. Moreover, $k_{\max } / k_{0} \simeq 0.28$ and 0.35 [the location of the growing peaks in Fig. 3(c) and $7(\mathrm{e})]$, corresponding to a beam deflection of 560 and $700 \mu \mathrm{m}$ after $2 \mathrm{~mm}$ of propagation, compare satisfactorily with Figs. $7(\mathrm{a})$ and $7(\mathrm{~d})$ for $T_{i}=300$ and $100 \mathrm{eV}$, respectively. Hence, our dispersion relations are validated quantitatively in the fluid formalism. The kinetic counterpart will be confronted to numerical studies in a future work.

\section{CONCLUSION}

The spatial growths of the filamentation and of the forward Brillouin instabilities of a RPP beam have been compared in the fluid and kinetic frameworks. Although the latter confirm the importance of spatial smoothing techniques on the control of the laser filamentation, this instability persists in the case of multi-ion or cold-enough plasmas. Albeit hydrodynamic codes predictions are correct regarding the description of the laser filamentation in a single-ion species plasma, they fail to capture the correct behavior in the multi-ion species case. Moreover, we also conclude that the use of Random phase plates does not guaranty stability of the laser propagation regarding FSBS. RPP beams can suffer large angle scatterings. Except for a single ion species plasma for moderate to large $Z_{i} T_{e} / T_{i}$-ratios, the FSBS growth can be imperfectly described in hydrodynamic codes leading to an ill prediction of the beam cone-angle increase and of the plasma smoothing. This brings to light the significance of the kinetic damping of driven acoustic waves able to affect the scattered spectrum.

During the calculation of the spatial growth of the forward instabilities, we neglected the transverse spatial laser envelope and its variations due to diffraction. Hence, our results should remain valid in realistic conditions for large-enough and flat-enough focal spots and for long enough focus. Moreover, our analytical results are averaged over the random phase plate elements thus failing to capture the corresponding statistical fluctuations.

The use of SSD, which causes the so-called speckles to vanish and change position during the interaction, is known experimentally to significantly stabilize the pump propagation [28]. The framework developed in this publication allows to include the spectral dispersion in the dispersion relations of importance for LMJ or NIF like facilities and is left for future work. Moreover, combining our spatial growth rates with our recent Monte-Carlo algorithm [34] opens the way to the description of the RPP forward Brillouin scattering in the vastly used ray tracing schemes, possibly greatly improving their predictions regarding high-energy laser experiments. For this end, the impact of a flow on the growth of the FSBS or the filamentation instability must be examined. Furthermore, a better understanding of the competition between the convective or absolute modes in the kinetic framework is required, as studied in Refs. [36, 38] for the fluid case (see Ref. [68] regarding the stimulated Raman scat- 
(a) FFT of transverse lineout

(b) Peak evolution
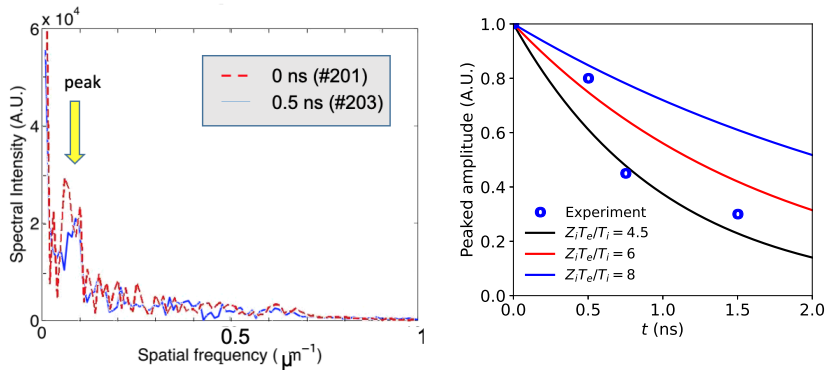

Figure A1. (a) Fourier transform of a central lineout across RCF similar as those shown in Fig. 5(b), unravelling a peak at the dominant wavelength. The two curves correspond to two different shots, taken at different times, as indicated in inset. (b) Temporal evolution of the peak amplitude (blue circles) observed in the Fourier transform identified by the yellow arrow in panel (a). Each point correspond to a different shot, where the time of the proton probing was changed with respect to the interaction laser propagating in the plasma [see examples of probing at two different times in Fig. 5(b)]. A decreasing exponential slope, $\exp (-\nu t)$, is plotted for a Landau damping frequency given by $\nu=\gamma_{0} c_{s} 2 \pi /[77 \mu \mathrm{m}]$ with the use of Eq. (6) for a $\mathrm{He}^{2+}$ plasma with $T_{e}=300 \mathrm{eV}$ [see Fig. 6(a)] and various $Z_{i} T_{e} / T_{i}$.

tering) or following Ref. [69]. Finally, the comparison of our model with NIF or LMJ-relevant experiments is currently underway.

\section{ACKNOWLEDGEMENTS}

We acknowledge important discussions with M. Grech and L. Gremillet. We acknowledge the expert support of the LULI technical teams. We also admit the role of the lockdown following the COVID19 plague for forcing us to take the time to finalize this theoretical work. This work has been done under the auspices of CEA-DAM and the simulations were performed using HPC resources at TGCC/CCRT and CEA-DAM/TERA.

\section{DATA AVAILABILITY}

The data that support the findings of this study are available from the corresponding authors upon reasonable request.

\section{APPENDIX: CONSTRAINING $Z_{i} T_{e} / T_{i}$ IN THE EXPERIMENT OF SEC. III C}

Figure A1(a) shows the Fourier transform of a RCF transverse lineout pointing out to a peak around a spatial frequency of $2 \pi / \lambda_{s} \sim 0.08 \mu \mathrm{m}^{-1}$, i.e. a wavelength of $\lambda_{s} \simeq 77 \mu \mathrm{m}$. Moreover, the comparison of the peak position at different times (blue plain line at $t=0 \mathrm{~ns}$ and red dashed line $t=0.5 \mathrm{~ns}$ ) demonstrates that the dominant wavelength remains mostly unchanged. In that condition, and noting that the probing proton deflection results from an electric field, the $\mathrm{RCF}$ dose modulation level depends mainly on the electron pressure fluctuations amplitude [70]. Hence, the peak temporal evolution of the experimental signal [blue circles in Fig. A1(b)] correlates with the damping of the lingering acoustic waves, previously triggered by the filamentation instability over the first nanosecond of the interaction beam. In this low density configuration $\left(n_{e} / n_{c} \simeq 10^{-2}\right)$, we may therefore directly compare this decrease with an exponential slope, $\delta n_{e} \propto \exp (-\nu t)$ where $\nu=\gamma_{0} c_{s} 2 \pi / \lambda_{s}$ is the Landau damping acoustic rate. The calculations for a $\mathrm{He}^{2+}$ plasma with $T_{e}=300 \mathrm{eV}$ [as suggested by Fig. 6(a)] and for three different values of $Z_{i} T_{e} / T_{i}$ illustrated in Fig. A1(b) demonstrate that $Z_{i} T_{e} / T_{i}=5$ reproduces correctly the experimental data. The experimental point at $t=0.5 \mathrm{~ns}$ is more consitent with the red curve which may suggest a value of $Z_{i} T_{e} / T_{i}$ larger than 5 earlier. Because of the available experimental data and the weak dependence of the filamentation growth rate on $Z_{i} T_{e} / T_{i}$, choice has been made to set the ratio to 5 for the analysis of Sec. III C.
[1] C. Cavailler, Plasma Physics and Controlled Fusion 47, B389 (2005).

[2] J. D. Lindl, P. Amendt, R. L. Berger, S. G. Glendinning, S. H. Glenzer, S. W. Haan, R. L. Kauffman, O. L. Landen, and L. J. Suter, Phys. Plasmas 11, 339 (2004).

[3] W. Zheng, X. Wei, Q. Zhu, F. Jing, D. Hu, X. Yuan, W. Dai, W. Zhou, F. Wang, D. Xu, X. Xie, B. Feng, Z. Peng, L. Guo, Y. Chen, X. Zhang, L. Liu, D. Lin, Z. Dang, Y. Xiang, R. Zhang, F. Wang, H. Jia, and X. Deng, Matter and Radiation at Extremes 2, 243 (2017).

[4] R. P. Drake, "Introduction to high-energy-density physics," in High-Energy-Density Physics: Fundamentals, Inertial Fusion, and Experimental Astrophysics, edited by L. Davison and Y. Horie (Springer Berlin Heidelberg, Berlin, Heidelberg, 2006) pp. 1-17.

[5] Y. R. Shen and N. Bloembergen, Phys. Rev. 137, A1787 (1965).

[6] D. W. Forslund, J. M. Kindel, and E. L. Lindman, Phys. Rev. Lett. 30, 739 (1973).

[7] Y. Kato, K. Mima, N. Miyanaga, S. Arinaga, Y. Kitagawa, M. Nakatsuka, and C. Yamanaka, Phys. Rev. Lett. 53, 1057 (1984).

[8] S. Skupsky, R. W. Short, T. Kessler, R. S. Craxton, S. Letzring, and J. M. Soures, J. Appl. Phys. 66, 3456 (1989).

[9] S. H. Glenzer, D. H. Froula, L. Divol, M. Dorr, R. L. Berger, S. Dixit, B. A. Hammel, C. Haynam, J. A. Hit- 
tinger, J. P. Holder, O. S. Jones, D. H. Kalantar, O. L. Landen, A. B. Langdon, S. Langer, B. J. MacGowan, A. J. Mackinnon, N. Meezan, E. I. Moses, C. Niemann, C. H. Still, L. J. Suter, R. J. Wallace, E. A. Williams, and B. K. F. Young, Nature Physics 3, 716 (2007).

[10] C. Labaune, Nature Physics 3, 680 (2007).

[11] P. Michel, Perte de cohérence d'un faisceau laser intense lors de la propagation dans un plasma, $\mathrm{Ph} . \mathrm{D}$. thesis (2003).

[12] P. Michel, C. Labaune, S. Weber, V. T. Tikhonchuk, G. Bonnaud, G. Riazuelo, and F. Walraet, Physics of Plasmas 10, 3545 (2003).

[13] P. M. Lushnikov and H. A. Rose, Plasma Physics and Controlled Fusion 48, 1501 (2006).

[14] G. Sarri, C. A. Cecchetti, R. Jung, P. Hobbs, S. James, J. Lockyear, R. M. Stevenson, D. Doria, D. J. Hoarty, O. Willi, and M. Borghesi, Phys. Rev. Lett. 106, 095001 (2011).

[15] D. E. Hinkel, E. A. Williams, and C. H. Still, Phys. Rev. Lett. 77, 1298 (1996).

[16] B. Bezzerides, Physics of Plasmas 5, 2712 (1998).

[17] P. E. Young, C. H. Still, D. E. Hinkel, W. L. Kruer, E. A. Williams, R. L. Berger, and K. G. Estabrook, Phys. Rev. Lett. 81, 1425 (1998).

[18] C. Ruyer, A. Debayle, P. Loiseau, M. Casanova, and P. E. Masson-Laborde, Physics of Plasmas 27, 102105 (2020).

[19] Z. J. Liu, S.-p. Zhu, L. H. Cao, C. Y. Zheng, X. T. He, and Y. Wang, Physics of Plasmas 16, 112703 (2009).

[20] L. Hao, Z. J. Liu, X. Y. Hu, and C. Y. Zheng, Laser and Particle Beams 31, 203-209 (2013).

[21] L. Hao, X. Y. Hu, C. Y. Zheng, B. Li, J. Xiang, and Z. J. Liu, Laser and Particle Beams 34, 270-275 (2016).

[22] D. F. DuBois, D. A. Russell, and H. A. Rose, Phys. Rev. Lett. 74, 3983 (1995).

[23] D. A. Russell and D. F. DuBois, Phys. Rev. Lett. 86, 428 (2001).

[24] C. Neuville, V. Tassin, D. Pesme, M.-C. Monteil, P.-E. Masson-Laborde, C. Baccou, P. Fremerye, F. Philippe, P. Seytor, D. Teychenné, W. Seka, J. Katz, R. Bahr, and S. Depierreux, Phys. Rev. Lett. 116, 235002 (2016).

[25] S. Depierreux, C. Neuville, C. Baccou, V. Tassin, M. Casanova, P.-E. Masson-Laborde, N. Borisenko, A. Orekhov, A. Colaitis, A. Debayle, G. Duchateau, A. Heron, S. Huller, P. Loiseau, P. Nicolaï, D. Pesme, C. Riconda, G. Tran, R. Bahr, J. Katz, C. Stoeckl, W. Seka, V. Tikhonchuk, and C. Labaune, Phys. Rev. Lett. 117, 235002 (2016).

[26] C. Neuville, C. Baccou, A. Debayle, P.-E. MassonLaborde, S. Hüller, M. Casanova, D. Marion, P. Loiseau, K. Glize, C. Labaune, and S. Depierreux, Phys. Rev. Lett. 117, 145001 (2016).

[27] C. Rousseaux, G. Huser, P. Loiseau, M. Casanova, E. Alozy, B. Villette, R. Wrobel, O. Henry, and D. Raffestin, Physics of Plasmas 22, 022706 (2015).

[28] R. L. Berger, B. F. Lasinski, A. B. Langdon, T. B. Kaiser, B. B. Afeyan, B. I. Cohen, C. H. Still, and E. A. Williams, Phys. Rev. Lett. 75, 1078 (1995).

[29] C. H. Still, R. L. Berger, A. B. Langdon, D. E. Hinkel, L. J. Suter, and E. A. Williams, Phys. Plasmas 7, 2023 (2000).

[30] P. Loiseau, O. Morice, D. Teychenné, M. Casanova, S. Hüller, and D. Pesme, Phys. Rev. Lett. 97, 205001 (2006).
[31] S. Hüller, P. E. Masson-Laborde, D. Pesme, M. Casanova, F. Detering, and A. Maximov, Phys. Plasmas 13, 022703 (2006).

[32] O. V. Batishchev, V. Y. Bychenkov, F. Detering, W. Rozmus, R. Sydora, C. E. Capjack, and V. N. Novikov, Physics of Plasmas 9, 2302 (2002), https://doi.org/10.1063/1.1461385.

[33] J.-L. Feugeas, P. Nicolaï, X. Ribeyre, G. Schurtz, V. Tikhonchuk, and M. Grech, Physics of Plasmas 15, 062701 (2008), https://doi.org/10.1063/1.2919791.

[34] A. Debayle, C. Ruyer, O. Morice, P.-E. Masson-Laborde, P. Loiseau, and D. Benisti, Physics of Plasmas 26, 092705 (2019).

[35] M. Duluc, D. Penninckx, P. Loiseau, G. Riazuelo, A. Bourgeade, A. Chatagnier, and E. d'Humières, Physics of Plasmas 26, 042707 (2019).

[36] M. Grech, Modifications des propriétés de cohérence des faisceaux laser dans les plasmas de fusion par confinement inertiel, Ph.D. thesis (2007).

[37] M. Grech, V. T. Tikhonchuk, G. Riazuelo, and S. Weber, Physics of Plasmas 13, 093104 (2006).

[38] M. Grech, G. Riazuelo, D. Pesme, S. Weber, and V. T. Tikhonchuk, Phys. Rev. Lett. 102, 155001 (2009).

[39] A. I. Akhiezer, I. A. Akhiezer, R. V. Polovin, A. G. Sitenko, and K. N. Stepanov, Plasma electrodynamics. Volume 2 - Non-linear theory and fluctuations (Pergamon Press, 1975).

[40] V. Bychenkov, W. Rozmus, A. Brantov, and V. Tikhonchuk, Phys. Plasmas 7, 1511 (2000).

[41] E. M. Epperlein, Phys. Rev. Lett. 65, 2145 (1990).

[42] R. L. Berger, E. J. Valeo, and S. Brunner, Physics of Plasmas 12, 062508 (2005).

[43] B. D. Fried, M. Gell-Mann, J. D. Jackson, and H. W. Wyld., J. Nuclear Energy: Part C 1, 190 (1960).

[44] J. F. Drake, P. K. Kaw, Y. C. Lee, G. Schmid, C. S. Liu, and M. N. Rosenbluth, The Physics of Fluids 17, 778 (1974).

[45] E. M. Epperlein, Physics of Plasmas 1, 109 (1994).

[46] V. Y. Bychenkov, J. Myatt, W. Rozmus, and V. T. Tikhonchuk, Physics of Plasmas 1, 2419 (1994).

[47] B. I. Cohen and C. E. Max, The Physics of Fluids 22, 1115 (1979).

[48] W. L. Kruer, The Physics Of Laser Plasma Interactions (Addison-Wesley Publishing Co., 1988).

[49] B. D. Fried, R. B. White, and T. K. Samec, The Physics of Fluids 14, 2388 (1971).

[50] E. A. Williams, R. L. Berger, R. P. Drake, A. M. Rubenchik, B. S. Bauer, D. D. Meyerhofer, A. C. Gaeris, and T. W. Johnston, Physics of Plasmas 2, 129 (1995).

[51] R. L. Berger, B. F. Lasinski, T. B. Kaiser, E. A. Williams, A. B. Langdon, and B. I. Cohen, Physics of Fluids B: Plasma Physics 5, 2243 (1993).

[52] A. J. Schmitt and B. B. Afeyan, Physics of Plasmas 5, 503 (1998).

[53] D. E. Hinkel, E. A. Williams, R. L. Berger, L. V. Powers, A. B. Langdon, and C. H. Still, Physics of Plasmas 5, 1887 (1998).

[54] J. Myatt, D. Pesme, S. Hüller, A. Maximov, W. Rozmus, and C. E. Capjack, Phys. Rev. Lett. 87, 255003 (2001).

[55] A. V. Maximov, I. G. Ourdev, D. Pesme, W. Rozmus, V. T. Tikhonchuk, and C. E. Capjack, Physics of Plasmas 8, 1319 (2001).

[56] A. J. Schmitt, The Physics of Fluids 31, 3079 (1988). 
[57] H. A. Rose and D. F. DuBois, Physics of Fluids B: Plasma Physics 5, 590 (1993).

[58] A. J. Mackinnon, P. K. Patel, R. P. Town, M. J. Edwards, T. Phillips, S. C. Lerner, D. W. Price, D. Hicks, M. H. Key, S. Hatchett, S. C. Wilks, M. Borghesi, L. Romagnani, S. Kar, T. Toncian, G. Pretzler, O. Willi, M. Koenig, E. Martinolli, S. Lepape, A. Benuzzi-Mounaix, P. Audebert, J. C. Gauthier, J. King, R. Snavely, R. R. Freeman, and T. Boehlly, Review of Scientific Instruments 75, 3531 (2004).

[59] R. A. Snavely, M. H. Key, S. P. Hatchett, T. E. Cowan, M. Roth, T. W. Phillips, M. A. Stoyer, E. A. Henry, T. C. Sangster, M. S. Singh, S. C. Wilks, A. MacKinnon, A. Offenberger, D. M. Pennington, K. Yasuike, A. B. Langdon, B. F. Lasinski, J. Johnson, M. D. Perry, and E. M. Campbell, Phys. Rev. Lett. 85, 2945 (2000).

[60] P. Bolton, M. Borghesi, C. Brenner, D. Carroll, C. De Martinis, F. Fiorini, A. Flacco, V. Floquet, J. Fuchs, P. Gallegos, D. Giove, J. Green, S. Green, B. Jones, D. Kirby, P. McKenna, D. Neely, F. Nuesslin, R. Prasad, S. Reinhardt, M. Roth, U. Schramm, G. Scott, S. TerAvetisyan, M. Tolley, G. Turchetti, and J. Wilkens, Physica Medica 30, 255 (2014).

[61] J. F. Ziegler, M. Ziegler, and J. Biersack, Nuclear Instruments and Methods in Physics Research Section B: Beam
Interactions with Materials and Atoms 268, 1818 (2010), 19th International Conference on Ion Beam Analysis.

[62] T. E. Cowan, J. Fuchs, H. Ruhl, A. Kemp, P. Audebert, M. Roth, R. Stephens, I. Barton, A. Blazevic, E. Brambrink, J. Cobble, J. Fernández, J.-C. Gauthier, M. Geissel, M. Hegelich, J. Kaae, S. Karsch, G. P. Le Sage, S. Letzring, M. Manclossi, S. Meyroneinc, A. Newkirk, H. Pépin, and N. Renard-LeGalloudec, Phys. Rev. Lett. 92, 204801 (2004).

[63] R. L. Berger, C. H. Still, E. A. Williams, and A. B. Langdon, Physics of Plasmas 5, 4337 (1998).

[64] H. A. Rose, Physics of Plasmas 3, 1709 (1996).

[65] P. E. Masson-Laborde, Modélisation réaliste de l'instabilité de diffusion brillouin stimulée., Ph.D. thesis (2010).

[66] P.E. Masson-Laborde, S. Hüller, D. Pesme, M. Casanova, P. Loiseau, and Ch. Labaune, J. Phys. IV France 133, 247 (2006).

[67] S. Hüller, P. E. Masson-Laborde, D. Pesme, C. Labaune, and H. Bandulet, Journal of Physics: Conference Series 112, 022031 (2008).

[68] T. Grismayer, A. Couairon, P. Mora, J.-C. Adam, and A. Héron, Physics of Plasmas 11, 4814 (2004).

[69] L. S. Hall and W. Heckrotte, Phys. Rev. 166, 120 (1968).

[70] N. L. Kugland, D. D. Ryutov, C. Plechaty, J. S. Ross, and H.-S. Park, Rev. Sci. Instrum. 83, 101301 (2012). 